Geo-Environmental Tactical Sensor Simulation (GEOTACS) Program

\title{
Defining Soil Materials for 3-D Models of the Near Surface: Preliminary Findings
}

Carla Roig-Silva, Benjamin D. Haugen, and Lillian D. Wakeley

March 2012

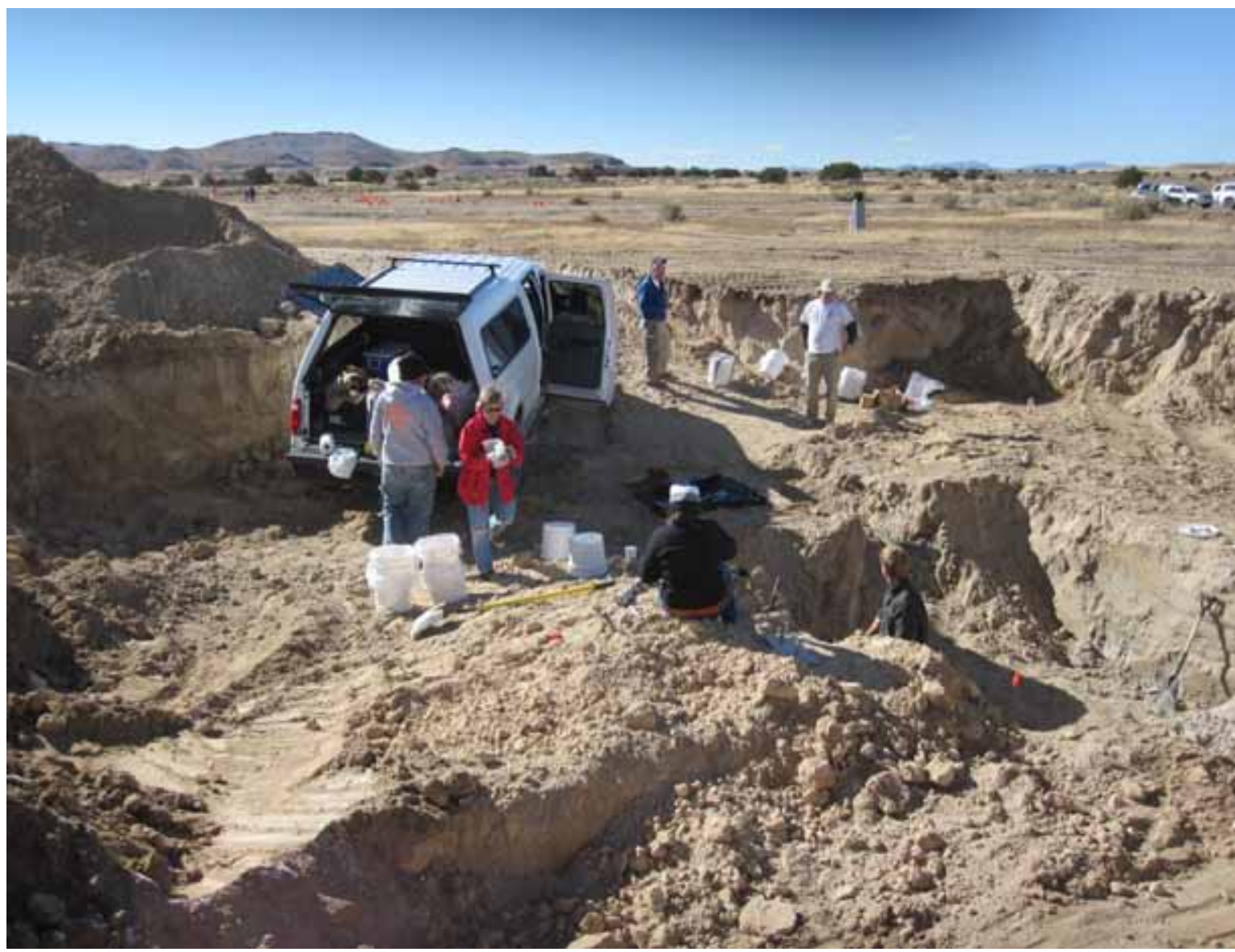




\section{Defining Soil Materials for 3-D Models of the Near Surface}

Carla Roig-Silva, Benjamin D. Haugen, and Lillian D. Wakeley

Geotechnical and Structures Laboratory

U.S. Army Engineer Research and Development Center

3909 Halls Ferry Road

Vicksburg, MS 39180-6199

Final report

Approved for public release; distribution is unlimited.

Prepared for U.S. Army Corps of Engineers

441 G Street NW

Washington, DC 20314-1000

Under Geo-environmental Tactical Sensor Simulation Program, ATO R.COE.2009.01 


\section{Abstract}

In support of three-dimensional modeling of soils in the near surface, a method was developed to define soil material types quantitatively using statistical comparisons of properties expected to impact sensor performance. To maintain technical continuity with other soils research and ensure relevance to soils engineering, many of the parameters chosen for statistical comparisons included conventional properties familiar to geotechnical researchers. Other, more sensor-specific soil properties, such as effective saturation and thermal conductivity, were used to allow for direct correlation between classified soil materials and sensor response. Initial trials of the method evaluated datasets from four sites in the U.S. and Asia. Early results showed that the number of statistically distinguishable materials tends to mimic the number of soil horizons sampled for a given dataset. Results also revealed a critical need for further research on the interactions of various soil properties and states between and among each other to determine the combinations that have the strongest influence on sensor response patterns. This protocol for delineating near-surface soil materials advances earlier techniques and improves the state-of-knowledge of modeling geologic features in three dimensions for sensor simulation.

DISCLAIMER: The contents of this report are not to be used for advertising, publication, or promotional purposes. Citation of trade names does not constitute an official endorsement or approval of the use of such commercial products. All product names and trademarks cited are the property of their respective owners. The findings of this report are not to be construed as an official Department of the Army position unless so designated by other authorized documents. 


\section{Contents}

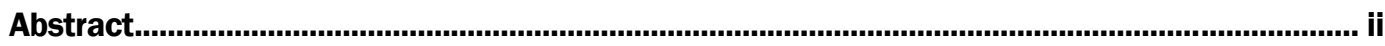

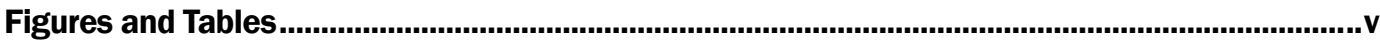

Preface ................................................................................................................................................vii

Unit Conversion Factors........................................................................................................ vifi

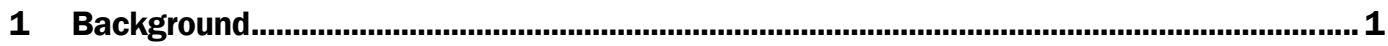

2 Soil Modeling for the ERDC Computational Testbed .............................................................. 2

2.1 Geo-environmental modeling for sensor simulation............................................ 2

2.2 The need to define material types for 3-D soil modeling ........................................ 3

2.3 TPROGS for GMS and subsurface complexity ...................................................... 5

3 Advancing the Method for Defining Soil Materials ................................................................ 7

3.1 Early work and mounting challenges............................................................... 7

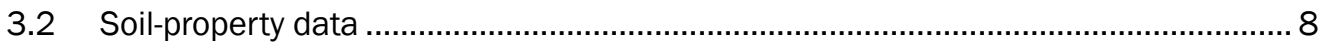

3.3 Development of a statistical approach............................................................... 9

3.4 Outline of steps to develop the new protocol .................................................... 10

4 Methods.............................................................................................................................13

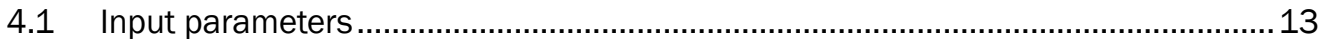

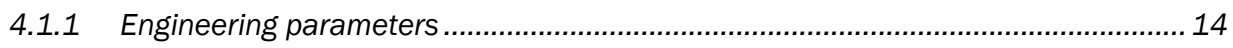

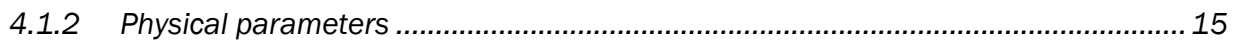

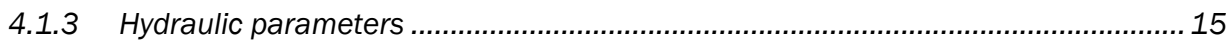

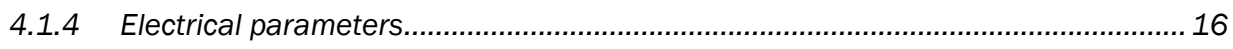

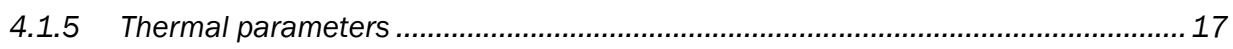

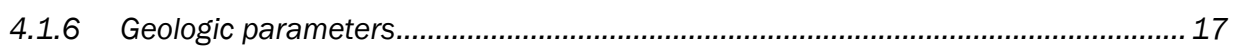

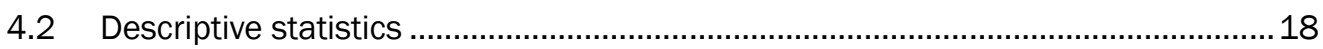

4.2.1 Soil parameter datasets .................................................................................. 18

4.2.2 Types of descriptive statistics.............................................................................. 19

4.2.3 Applications of descriptive statistics..................................................................... 20

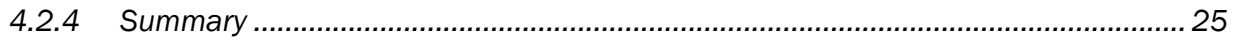

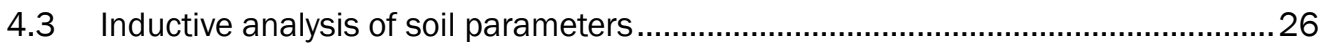

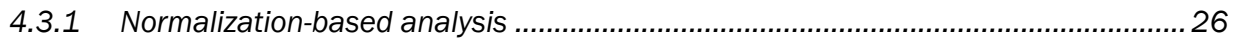

4.3.2 Comparisons of normalized values ......................................................................... 27

4.4 Application of the protocol ............................................................................... 28

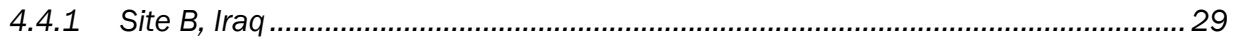

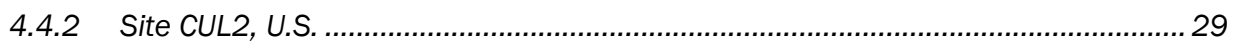

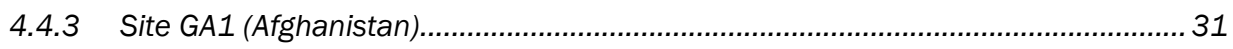

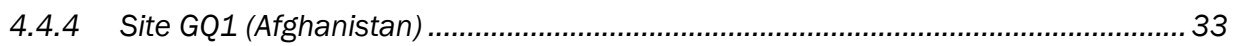


5.1 Quantitative versus qualitative analysis.......................................................... 34

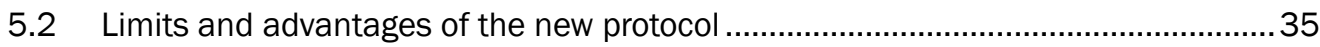

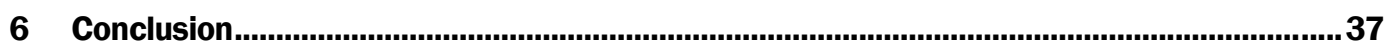

6.1 Adaptable improvements on previous methods ............................................. 37

6.2 Quantitative soil parameter comparisons ..............................................................3

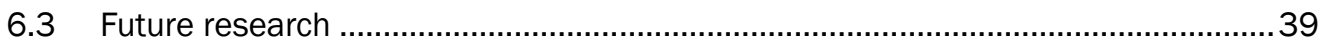

References.................................................................................................................................................40

Appendix A: Raw Values of Soil Parameters at Each Protocol Test Site.....................................44

Report Documentation Page 


\section{Figures and Tables}

\section{Figures}

Figure 1. Schematic diagram of parameters used in the development of the protocol. Red boxes indicate commonly measured properties. Blue boxes indicate less commonly measured properties that were chosen for their impact on sensor response. Green boxes are geologic parameters chosen because of their controlling influence on other parameters. Parameters in italics are qualitative. Parameters in plain text are quantitative.

Figure 2. Probability plots for quantitative parameters. Blue lines represent the 95 percent confidence interval for a normal distribution. As shown by the data values for the parameters (red dots), all parameters have a non-normal distribution.

Figure 3. Summary statistics for the coefficient of uniformity $(\mathrm{Cu})$. The histogram on top shows the data being strongly negatively-skewed. Histogram is leptokurtic. The corresponding box plot and 95 percent confidence intervals for mean and median are below histogram.

Figure 4. Summary statistics for the coefficient of curvature (Cc). The histogram on top shows the data being strongly negatively-skewed. Histogram is leptokurtic. The corresponding box plot and 95 percent confidence intervals for mean and median are below histogram.

Figure 5. Summary statistics for the effective saturation (Se). The histogram on top shows the data being negatively-skewed. Histogram is platykurtic. The corresponding box plot and 95 percent confidence intervals for mean and median are below histogram.

Figure 6. Summary statistics for porosity $(\varphi)$. The histogram on top shows the data being strongly negatively-skewed. Histogram is leptokurtic. The corresponding box plot and 95 percent confidence intervals for mean and median are below histogram

Figure 7. Summary statistics dry bulk density $(\rho)$. The histogram on top shows the data being strongly positively-skewed. Histogram is leptokurtic. The corresponding box plot and 95 percent confidence intervals for mean and median are below histogram.

Figure 8. Summary statistics for specific conductance ( $\sigma$ ). The histogram on top shows the data being strongly negatively-skewed. Histogram is leptokurtic. The corresponding box plot and 95 percent confidence intervals for mean and median are below histogram.

Figure 9. Summary statistics for thermal resistivity $(R)$. The histogram on top shows the data being strongly negatively-skewed. Histogram is leptokurtic. The corresponding box plot and 95 percent confidence intervals for mean and median are below histogram.

Figure 10. Summary statistics for thermal diffusivity $(\alpha)$. The histogram on top shows the data being strongly Inegatively-skewed. Histogram is leptokurtic. The corresponding box plot and 95 percent confidence intervals for mean and median are below histogram.

\section{Tables}

Table 1. Types of data used in early subsurface modeling efforts for the GEOTACS program. 5

Table 2. Skewness descriptive terminology. ............................................................................... 19

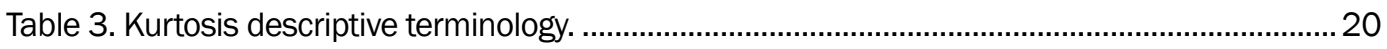


Table 4. Summary for descriptive statistics of quantitative parameters. $C_{u}=$ coefficient of uniformity; $C_{c}=$ coefficient of curvature; $S e=$ effective saturation; $\varphi=$ porosity; $\rho=$ dry bulk density; $\sigma=$ specific conductance; $R=$ thermal resistivity; $\alpha=$ thermal diffusivity.

Table 5. Example of using Z-score normalization to reveal relationships between data values at depth. Colors represent individual material types. Standard values at depths 100 to $140 \mathrm{~cm}$ (relative to all depths) showed a difference of < 0.50; thus, they were grouped into one material type based only on the dry-bulk-density parameter. Density values were missing in the upper $60 \mathrm{~cm}$ at this site.

Table 6. Standardized values for $\mathrm{CBU}$ site. Colors by column represent groups where there is an absolute Z-score difference of less than 0.50. Sample= sampling ID and depth in $\mathrm{cm}$; $\mathrm{Std}=$ standardized value; USCS $=$ Unified Soil Classification System; $\mathrm{C}_{\mathrm{u}}=$ coefficient of uniformity; $\mathrm{C}_{\mathrm{c}}=$ coefficient of curvature; $\mathrm{Se}=$ effective saturation; $\varphi=$ porosity; $\rho=$ dry bulk density; $\sigma=$ specific conductance; $\mathrm{R}=$ thermal resistivity; $\alpha=$ thermal diffusivity; $Q t z=q u a r t z ;$ Alb=albite; Ksp=Potassium-rich feldspars; Clay=total clay mineral content; Carb=carbonate.

Table 7. Final definition of material types for the Iraq site.

Table 8. Standardized values for U.S. site. Colors by column represent groups where there is an absolute Z-score difference of less than 0.50. Sample= sampling ID and depth in $\mathrm{cm}$; Std=standardized value; USCS = Unified Soil Classification System; $\mathrm{C}_{\mathrm{u}}=$ coefficient of uniformity; $\mathrm{C}_{\mathrm{c}}=$ coefficient of curvature; $\mathrm{Se}=$ effective saturation; $\varphi=$ porosity; $\rho=$ dry bulk density; $\mathrm{R}=$ thermal resistivity; $\alpha=$ thermal diffusivity.

Table 9. Final definition of material types for the U.S. site.

Table 10. Standardized values for GA1 site. Colors by column represent groups where there is an absolute Z-score difference of less than 0.50. Sample= sampling ID and depth in $\mathrm{cm}$; $\mathrm{Std}=$ standardized value; USCS $=$ Unified Soil Classification System; $\mathrm{Cu}_{\mathrm{u}}=$ coefficient of uniformity; $C_{c}=$ coefficient of curvature; $S e=$ effective saturation; $\varphi=$ porosity; $\rho=$ dry bulk density; $R=$ thermal resistivity; $\alpha=$ thermal diffusivity.

Table 11. Final definition of material types for site GA1

Table 12. Standardized values for GQ1 site. Colors by column represent groups where there is an absolute Z-score difference of less than 0.50. Sample= sampling ID and depth in $\mathrm{cm}$; $\mathrm{Std}=$ standardized value; USCS= Unified Soil Classification System; $\mathrm{C}_{\mathrm{u}}=$ coefficient of uniformity; $\mathrm{C}_{\mathrm{c}}=$ coefficient of curvature; $\mathrm{Se}=$ effective saturation; $\varphi=$ porosity; $\rho=$ dry bulk density; $R=$ thermal resistivity; $\alpha=$ thermal diffusivity.

Table 13. Final definition of material types for site GQ1. 


\section{Preface}

This study was conducted for the military direct research Geoenvironmental Tactical Sensor Simulation (GEOTACS) Program under Army Technical Objective (ATO) R.COE2009.06. Dr. David Horner was the ATO Manager, Josh R. Fairley was the GEOTACS Program Manager, and Dr. Niki Goerger was the Technology Staff Officer.

The work was performed by personnel with the Geotechnical Engineering and Geosciences Branch (GEEB), Geosciences and Structures Division (GSD) and the Research Group, Engineering Systems and Materials Division (ESMD), all with the Geotechnical and Structures Laboratory (GSL), U.S. Army Engineer Research and Development Center (ERDC). The researchers were under the general supervision of Dr. Monte L. Pearson, Chief, GEEB; Bartley P. Durst, Chief, GSD; Dr. Larry N. Lynch, Chief, ESMD; Dr. William P. Grogan, Deputy Director, GSL; and Dr. David W. Pittman, Director, GSL.

COL Kevin J. Wilson was the Commander and Executive Director of ERDC. Dr. Jeffery P. Holland was the Director. 


\section{Unit Conversion Factors}

\begin{tabular}{|l|c|l|}
\hline Multiply & \multicolumn{1}{l|}{ By } & To Obtain \\
\hline feet & 0.3048 & meters \\
\hline inches & 0.0254 & meters \\
\hline miles (U.S. statute) & $1,609.347$ & meters \\
\hline pounds (mass) & 0.45359237 & kilograms \\
\hline pounds (mass) per cubic foot & 16.01846 & kilograms per cubic meter \\
\hline pounds (mass) per cubic inch & $2.757990 \mathrm{E}+04$ & kilograms per cubic meter \\
\hline square feet & 0.09290304 & square meters \\
\hline square yards & 0.8361274 & square meters \\
\hline tons (force) per square foot & 95.76052 & kilopascals \\
\hline yards & 0.9144 & meters \\
\hline
\end{tabular}




\section{Background}

The development of a three-dimensional (3-D) geologic model is a multistep process. One of the first steps is to define a representation of the geologic framework that controls the spatial distribution of soil properties in the model (Turner et al. 2007). The distribution and variability of soil properties in the shallow subsurface are controlled by the geologic architecture, which is the size, shape, and arrangement of soil features attributable to the geologic origin of soils in an area (Wakeley et al. 2010). The geologic architecture - and therefore the distribution of soil properties - results from regional and local geologic processes that deposited or formed the soil (Weissmann 1999) and subsequent human activity and natural chemical and physical processes that later modified it. The research described in this report was executed to develop a comprehensive and adaptable method for delineating soil materials at multiple scales based upon soil properties in the shallow subsurface.

The purpose of the Geo-environmental Tactical Simulation (GEOTACS) program is to develop comprehensive, high-fidelity models that will predict and improve performance of sensor systems for surface and near-surface target detection in complex geo-environmental settings (Horner 2009; Fairley et al. 2010). The intent of GEOTACS research is to improve the realism of representations of the geo-environment. Rather than developing a better sensor to find a needle (the target) in a haystack (the geoenvironment), the GEOTACS program focuses on better characterization and representation of the haystack to improve automated "needle"recognition algorithms with fewer false alarms. Realistic 3-D geologic models must be included in the comprehensive GEOTACS models. This research is a step toward improving the realism of soil property distributions in 3-D geologic models. 


\section{Soil Modeling for the ERDC Computational Testbed}

\subsection{Geo-environmental modeling for sensor simulation}

Since 2005, ERDC researchers have been developing a computational testbed to explore near-surface process interaction at a fine scale to better understand the phenomena behind the interaction of sensors and soils (Eslinger et al. 2007; Fairley et al. 2010; Howington et al. 2010; Peters et al. 2007). To support the computational testbed, the GEOTACS subsurface realizations team developed 3-D geologic conceptual models of the nearsurface using the Department of Defense Groundwater Modeling System (GMS) platform (Wakeley et al. 2010, 2011). Various types of soil data from several areas of interest (AOI) were assembled in GMS to construct 3-D geologic models that were consistent with geologic architecture. A transition-probability geostatistics package - TPROGS for GMS - was used to generate multiple static models of soil materials' distributions at depth. Each soil material had a unique set of properties that was determined using statistical analyses of soil properties (see Chapters 3 and 4).

Each static model, or realization, is a possible representation of reality. Using spatial constraints provided by the modeler and statistical parameters in the software module, TPROGS for GMS generates the geologic architecture (i.e., distribution of soil materials) in each realization. Unlike many other geologic modeling methods, TPROGS does not directly emulate the input borehole data. Instead, it distributes the soil materials according to the probability that any two or more materials may be found juxtaposed, and where they occur most frequently within the model grid. The borehole data are used only to calculate the distribution values, not to dictate where the materials will be placed at the location of the borehole. This facet of TPROGS is what enables the production of multiple realizations that have the same statistical probability of existing, given the available data.

In the voids between the specified locations, the soil materials may be distributed differently in each realization, but the statistical probability that any one material exists in a given place will always remain the same. That is, TPROGS fills the voids in each realization based on the probabilities of materials being juxtaposed to one another, given the input data. This results 
in a series of physically different but statistically identical individual static models (or realizations).

The limitations of the data are such that having a model that precisely replicates the distribution defined at the location of each borehole does not provide any advantages in this application. Many of the boreholes are defined by expert judgment and, thus, do not necessarily reflect the true material distribution at that point. Several of the boreholes are from recreations of real data, but these are only a small part of the total number of boreholes.

The boreholes are also sparsely distributed throughout the model. Even if every borehole in the model were "real" at the scale of interest, a great deal of interpretation would be required to fill in the void. Despite not being an exact reproduction of the borehole data, a TPROGS model grid captures the spatial tendencies of the data-- such as pinching, abrupt material transitions, and space-variant volume changes-- that are the most important considerations in modeling geologic architectures of soil materials. Because they are equally likely to be an accurate representation of reality, any of the multiple realizations, then, can be used as the subsurface component of a full geo-environmental model.

The 3-D geologic models created in this manner thus provide a framework for distributing material properties in the subsurface and in so doing directly support production of more complex testbed models. Howington et al. (2010) explain the ERDC computational testbed, of which 3-D geologic models of near-surface soils are a critical component.

\subsection{The need to define material types for 3-D soil modeling}

Soils properties affect sensor response, and sensors used for target recognition rely on differences in properties among materials and between a given material and an encapsulated target to detect threats (Fairley et al. 2010; Howington et al. 2010; Howington et al. 2011). Thus, to model the response of a sensor to various subsurface conditions, it is necessary to have a scientifically sound and reproducible method to delineate multiple subsurface material types. The method must be applicable to a variety of conditions and sensors at many different scales. Moreover, the properties considered in the development of the method, or protocol, must be relevant to the sensors being used. Some commonly measured soil 
properties have little bearing on the response of sensor modalities, while others have a significant impact.

Soil properties used in the Unified Soil Classification System (USCS) (U.S. Army Engineer Waterways Experiment Station 1960) are among the most commonly measured and reported for engineering purposes. The soil classes of the USCS are inadequate for defining soil material types in a way that is meaningful for sensor systems. To understand why the USCS is inadequate, consider that two soil samples from one site may meet the grain-size requirements of a single USCS class but at the same time have very different densities, moisture contents, and saturated permeabilities. Because moisture content, density, and saturated permeability are known to affect sensor response (Andraski 1996; Boadu 2000; Curtis 2001; Koh and Wakeley 2010), the two soils that would be considered a single material in the USCS may impact a given sensor modality in very different ways. Thus, the method used to define soils materials for GEOTACS modeling must be based on properties that are expected to impact sensor response rather than on soil classes alone.

Other commonly used soil classification systems, such as the U.S. Department of Agriculture (USDA) Soil Taxonomy (Soil Survey Staff 2006) and the American Association of State Highway and Transportation Officials (AASHTO) system (2010) are also insufficient for sensor-response modeling. Similar to the USCS, these methods use a set of soil properties useful in a limited number of applications. Despite their limited purview, however, they do indicate an important aspect of soil classification: specific applications require specific classification systems. Thus, when the application is 3-D modeling of the subsurface for sensor response simulations, an appropriate classification system must be developed and used.

In early GEOTACS models, subsurface materials were distinguished from each other by considering a suite of soil properties expected or known to affect sensor response. Both descriptive and quantitative data were considered. Table 1 contains a list of the data types used in early subsurface models.

Early determinations of soil materials at the GEOTACS modeling sites were made using a combination of expert geologic judgment and simple statistical analyses of the properties in Table 1 (Wakeley et al. 2011). The aim of this effort was to create a 3-D geologic model that was an accurate 
Table 1. Types of data used in early subsurface modeling efforts for the GEOTACS program.

\begin{tabular}{|l|l|}
\hline Quantitative data types & Qualitative data types \\
\hline Mineral composition & Geomorphic setting from imagery \\
\hline $\begin{array}{l}\text { Percentages of fine particles (clay and silt), } \\
\text { sand, and gravel }\end{array}$ & $\begin{array}{l}\text { Geologic setting and descriptions of soil types } \\
\text { from field notes }\end{array}$ \\
\hline Dry bulk density & USCS classification and soil texture \\
\hline Plasticity index & \\
\hline Gravimetric and volumetric water content & \\
\hline Saturated hydraulic conductivity & \\
\hline Cation exchange capacity & \\
\hline
\end{tabular}

representation of what a sensor might actually "see" in the subsurface, rather than to create a model that showed only geologic features. This method was successful but revealed a need to develop more robust quantitative tools for defining material types. Chapters 3 and 4 of this report describe in detail the new, more robust method that was developed.

\subsection{TPROGS for GMS and subsurface complexity}

TPROGS for GMS was used to generate multiple static models of the distribution of material types at depth. GMS was chosen as the platform because it was successfully used to generate complex 3-D conceptual geologic models in previous work (Talbot et al. 2003; Wakeley et al. 2007). The value of TPROGS is in its assumptions. Because it uses advanced statistical methods to "fill in the gaps" in geologic data, it is an excellent choice for modeling geologic features where data are sparse.

The current version of TPROGS for GMS is limited to producing realizations that contain no more than five material types. These five materials are essentially five types of soil, each with a characteristic set of properties. Five materials are sufficient in some situations but may be insufficient in others, depending on the scale of interest and the geological fidelity required. In other words, areas with complex geology may require more than five materials to represent accurately the variability in soil properties that can be detected by a given sensor. Likewise, areas with simple geology may require fewer than five material types if the model is developed at a relatively coarse scale.

Soil variability from one geo-environment to another poses an additional problem: the complexities of material distribution can confound traditional 
"layer-cake" 3-D geologic models. In real environments, distinct soil materials can be laterally and vertically intermittent. Layer pinching gradational changes at one location, abrupt contacts at another, multiple overlapping units of the same material, and other unique features of real environments can preclude the use of "layer-cake" models. TPROGS is one of the best solutions to this problem developed to date. Nonetheless, a limited number of material types will always require a robust method to determine the criteria used to distinguish one soil material from another. Without such a method, an accurate model of the near-surface relevant to sensor response can never be developed. The combination of the soil material definition protocol described hereafter and the relatively simple geologic conditions of the areas of interest allowed more realistic and accurate models to be produced using TPROGS for GMS. 


\section{Advancing the Method for Defining Soil Materials}

\subsection{Early work and mounting challenges}

While early work at developing a protocol for defining soil materials (described in Chapter 2) was sufficient for the need at the time, additional work was required to meet advancing GEOTACS program goals. Recent work focused on the development of a protocol that is both more quantitative and more adaptable. The new protocol described in the remainder of this report is the first iteration of a tool that can be used to delineate soil materials at multiple scales, in multiple geologic settings, and with wide-ranging applicability to simulations of sensor response. This tool is the first of its kind and provides a starting point for future sensor simulations, soil-property modeling, and 3-D geologic modeling.

Similar to previous work, the protocol provides decision points at which expert judgment is critical to the modeling effort. More robust quantitative methods in the new protocol make it more versatile, more reproducible, and simpler to implement. Ultimately, the protocol detailed in this report will improve the accuracy and realism of 3-D models of soil properties at the scale required for the GEOTACS Program - about 2 to $4 \mathrm{~cm}$ (Eslinger et al. 2007).

The greatest challenge to advancing the protocol is one of numbers. The various properties known to affect sensor response must be measured, compared, and analyzed, such that the difference between soil materials is clearly defined in a reproducible manner. This challenge is complicated by correlations between the properties (e.g., density and water content), their differing effects on sensor systems, and their various scales and units of measurement. Another great challenge is the lack of precedent: no soilclassification system addresses the specific needs of sensor response simulations. Because of these challenges, the subsurface realizations team had to develop a novel approach to the problem within a limited research scope.

In its current form, the protocol for defining material types is tailored to the requirements of the GEOTACS research program. Yet part of the premise of 
this method is that it provides a tactical framework for approaching definition for materials for 3-D geologic models. That is, rather than requiring a certain set of soil properties and/or environments to be applicable, it can be adapted to any set of material properties for almost any purpose. The protocol could, for example, be used to define materials by their effects on blast response or by their effects on geophysical sensors used to detect subsurface anomalies. The protocol allows the user to define materials that are consistent with the geologic architecture of a site by analyzing properties known to impact the response of the sensor or the system of interest.

The protocol also includes more conventional engineering properties to maintain continuity for users who are familiar with the USCS soil classification system. With respect to the GEOTACS Program, the results of material definitions using the protocol are used in the context of imagery, geologic data, and field descriptions to develop the geologic architecture of the site being modeled. The protocol is a foundational component of the mission of the subsurface realizations team.

\subsection{Soil-property data}

To produce most 3-D geologic models, high-density datasets are input into a modeling program, and the program "connects the dots." This method works well where data are widely available or easily obtainable but presents a problem in regions that are difficult to access or otherwise data limited. Detailed data collection is impractical for the GEOTACS Program because of the many logistical limitations in active military operations zones and locations under military scrutiny. Thus, the TPROGS for GMS makes possible the required modeling component by using sparse proxy data analyzed using the protocol presented herein to assign material types to the soils and distribute those materials "virtually" through the subsurface using TPROGS for GMS.

The data available for this study came from 20 sites in arid environments in the U.S., Iraq, and Afghanistan. Data were derived from soil samples collected by ERDC research teams using a standardized sampling method (Wakeley et al. 2009). At each site, undisturbed soil samples were collected at standard depth intervals from trenches up to $2 \mathrm{~m}$ deep using 3 -in.-diameter hollow cylinders. The cylinders were capped and sealed in the field to preserve in-situ density, particle geometry, and moisture content. Depending on trench depth and local geology, the depth intervals 
between soil samples varied from 10 to $20 \mathrm{~cm}$. By using a shovel or a pick, the research team collected additional soil samples at locations within and outside the trench, as needed, to support interpretations of local geologic architecture.

Physical, mechanical, chemical, and electrical properties of each soil sample were measured either by the ERDC laboratories in Vicksburg, MS, or by the laboratories of Daniel B. Stephens and Associates in Albuquerque, NM, or by both. Soil-properties data were organized by sample site and depth and are continuously maintained in a database at the ERDC. Not every desired soil property could be measured at every site and depth range. In some cases, field conditions were not ideal for sampling, and testable samples were not obtained. As with any large sampling program, other logistical and processing issues led to gaps in the soil properties datasets. These gaps made it even more imperative that the protocol and distribution of resulting materials work well with limited datasets.

\subsection{Development of a statistical approach}

The development of a new soil classification method was motivated by two factors. First, it is essential to be able to compare soil samples from multiple sites and depth ranges, considering that a multitude of soil properties influence sensor response. Furthermore, the volume of soils data available albeit not concentrated in any one location - precluded the use of simple qualitative methods. A robust mathematical approach that could handle the types and diversity of available data was needed. Second, it was imperative that the individual soil materials identified for use in GMS modeling efforts had consistent properties between and among all models produced.

In earlier 3-D modeling work, the soil materials were defined by combining expert geologic architecture interpretations with visual comparisons of soil property data and some simple statistical averaging. This approach was adequate to produce an initial set of 3-D model realizations but was not sufficiently reproducible. It relied heavily on expert judgment. Although expert judgment is an important aspect of determining the constraints of any geologic model, it is ultimately insufficient for true pattern recognition and can be inconsistent between individual experts.

The protocol detailed here is the next step toward developing a system of combined quantitative and qualitative tools that are specifically designed to address the known relationships between certain soil properties and 
sensor responses. It is based on a reproducible set of processes and is less open for interpretation than previous methods.

\subsection{Outline of steps to develop the new protocol}

The first step in developing our approach was to determine the set of soil properties that would be used in the protocol. Multiple soil properties also called "parameters" in this method - had already been measured in both the field and the laboratory. This set was, thus, the first constraint on our choices: we limited the parameters to those in the dataset. This was reasonable, since each parameter was measured specifically because it was known to impact sensor response (Wakeley et al. 2009). That is, we could be reasonably sure that each of the soil properties in the dataset was important to our mission. The set of parameters chosen for this protocol is described in Chapter 4.

The next and perhaps most challenging part of our mission was to find a statistical method that could be used to compare multiple properties from multiple sites and depth ranges. This challenge was complicated by the fact that soil properties vary at many scales and in many dimensions (vertically and laterally), both within one material and between materials and at the very small and very large scales.

A further complication was that most soil properties are measured in unique units, and the range of possible values for each property can vary an order of magnitude or more. Before we could address our goal of determining the best statistical method to compare the individual data points (i.e., a soil property at a certain depth and location), we first had to determine how to standardize the data so that soil properties could be compared with one another. This challenge required us to develop an understanding of the data we had using descriptive statistics and to determine the best method to correct for any data characteristics that could curtail later statistical analysis.

Descriptive statistics--including probability plots, box plots of means and clustered values, and normality tests--were used to understand the characteristic features of available data. These statistical tools indicate how the data are distributed (e.g., Gaussian vs. non-Gaussian, variance around the mean, etc.). They also show evidence for consistent patterns in the data such as skewness and kurtosis (Mathews 2005). Once the descriptive statistics were run on each dataset, we determined the most feasible 
normalization approach. This decision was based on many factors-including its distribution, variance, skewness, and size. This process is described in more detail in Chapter 4.

After normalizing the data, we still had to decide how to compare them. This was a complicated process that raised several questions. First, for any two soil materials, what threshold in property difference is sufficient for defining them as separate materials, such that a sensor can "see" the difference between them? Second, what correlations, if any, exist between individual soils properties? Finally, is there some weighting factor or factors that need to be applied to certain properties to compare them?

The first question was beyond the scope of this research, and no answer was found in the literature. It was not critical to determine the property difference threshold before developing the protocol: A threshold value can be chosen without altering the protocol itself. However, to fully test the new protocol, it is critical that the question be answered because the answer will be needed for subsequent subsurface modeling efforts.

The second question defines a gap in sensor physics research. Most sensor systems use varying degrees of electromagnetic (EM) energy to penetrate materials and detect foreign objects. Research on the effect of soil properties on sensor systems has focused on moisture content (Curtis 2001; Koh and Wakeley 2010; van Dam et al. 2005). Few other properties have been considered as important, and clear relationships between and among the various soil properties have not been defined (Moysey and Knight 2004; Rechenmacher and Medina-Cetina 2007; van Dam et al. 2005). During the development of this protocol, we were able to determine that certain properties are statistically correlated. Often, the observed correlations are due to the fact that certain properties are derivatives of others. These "collinear" properties were removed from the protocol because they would increase the "weight" of a single soil property (i.e., the property from which another, collinear property was derived) relative to the others.

Using empirical substitutions and the information provided by our search for answers to the first two questions, we answered the final question. Using the generic threshold value and the shortened list of properties developed by attempting to answer the first two questions above, we created a simple but effective scheme to delineate individual material layers in the shallow subsurface. Values of a certain soil property at a certain depth and site are 
compared to each other based on the threshold. A difference in parameter values at two points in space that exceeds the threshold means that they are distinct materials; a difference in values less than the threshold means that they are the same.

Once the comparisons of soil parameters were completed for all depth ranges at a site, layers of soil materials were defined by majority. For example, if the majority of the properties indicated that the first $20 \mathrm{~cm}$ of soil was a single material, it was then assigned a corresponding material identifier (ID). Likewise, if the next depth range $(20-40 \mathrm{~cm})$ had some soil properties that indicated it was the same material as the first $20 \mathrm{~cm}$, but most properties indicated that it was different, the $20-$ to $40-\mathrm{cm}$ range was assigned a different, unique material ID. In a tie, the most heavily "weighted" soil properties were used to define the material IDs. In this way, through the use of a series of soil properties, individual soil materials could be identified at multiple depths and at multiple sites within an AOI. 


\section{Methods}

This chapter discusses the input parameters used in the protocol and the reasons they were selected. It also includes descriptive statistics of the parameters and plots of the datasets where data from multiple sites were treated as a single dataset.

\subsection{Input parameters}

Nine quantitative and two qualitative soil parameters are evaluated in the protocol (Figure 1). Some of the parameters-such as USCS soil classification, effective saturation, and dry bulk density-- are commonly acquired for engineering purposes. Other properties are less commonly measured but are considered important when determining how a sensor will respond to a given material. Examples include effective saturation, thermal diffusivity, and specific conductance (Howington et al. 2011). Engineering and physical parameters (Figure 1) are included in the protocol because they are most familiar to soil scientists and engineers and because they are part of standard practice in soil classification. Hydraulic, electrical, and thermal properties are included because of their influence on sensor response.

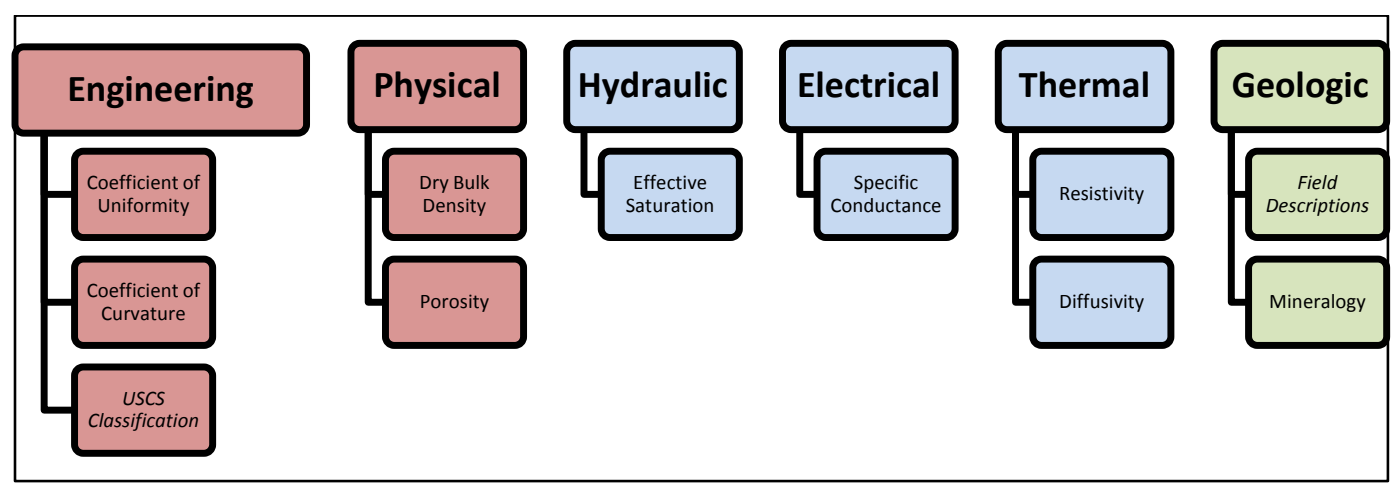

Figure 1. Schematic diagram of parameters used in the development of the protocol. Red boxes indicate commonly measured properties. Blue boxes indicate less commonly measured properties that were chosen for their impact on sensor response. Green boxes are geologic parameters chosen because of their controlling influence on other parameters. Parameters in italics are qualitative. Parameters in plain text are quantitative.

All of the aforementioned properties are controlled to some degree by geologic conditions. Mineralogy has a direct impact on the performance and capability of some sensors. Geologic parameters are thus included to provide context for the evaluation and analysis of the other parameters and to account for the impacts of mineral composition on sensor response. 


\subsubsection{Engineering parameters}

The coefficient of uniformity ( $C_{u}$; Equation 1)and coefficient of curvature $\left(C_{c}\right.$; Equation 2)are parameters used in standard engineering practice (U.S. Army 1960; ASTM Standard D2487-10, 2010) to classify coarsegrained soils (more than 50 percent of the material sand size and coarser). Each coefficient is calculated from grain-size analyses as part of the USCS process. These parameters define the grading of a soil, which gives an indication of other engineering properties, such as shear strength.

$$
C_{u}=\frac{D_{60}}{D_{10}}
$$

and

$$
C_{c}=\frac{\left(D_{30}\right)^{2}}{\left(D_{10}\right)\left(D_{60}\right)}
$$

where:

$D_{10}=$ grain size $(\mathrm{mm})$ where 10 percent of grains are smaller than the $\mathrm{D}_{10}$;

$D_{30}=$ grain size $(\mathrm{mm})$ where 30 percent of grains are smaller than the $\mathrm{D}_{30}$;

$D_{60}=$ grain size $(\mathrm{mm})$ where 60 percent of grains are smaller than the $\mathrm{D}_{60}$.

The coefficient of uniformity is a measure of the range of particle sizes in a given sample; the higher the number, the wider the range of particle sizes. However, $C_{u}$ does not reveal what those particle sizes are. Two soils with vastly different $D_{60}$ and $D_{10}$ values could have the same $C_{u}$. On the other hand, the coefficient of curvature indicates how evenly distributed particle sizes are within the total range of particle sizes present in a sample. That is, $C_{c}$ reveals the difference between a soil that has only boulders in uniformly sandy matrix and a soil that has an even distribution of grain sizes from boulders to gravel to sand (where $C_{u}$ may not). When used together, the $C_{c}$ and $C_{u}$ parameters define the soil gradation and particle size uniformity of a given sample. These properties are not known to directly affect sensor response, but they have been related to permittivity 
and conductivity of soils, which can impact sensors (Thevanayagan 1995; van Dam et al. 2005).

In soil engineering, $C_{u}$ and $C_{c}$ are considered meaningful only for classifying coarse-grained soils, such as clean gravels (USCS classes GW or GP) and clean sands (USCS classes SW or SP) that have more than 50 percent of material larger than a No. 200 sieve $(0.075 \mathrm{~mm})$ and less than five percent fines (silt-size and clay-size particles). Atterberg limits are the standard method for distinguishing finer-grained soils. However, for this protocol, we considered the $C_{u}$ and $C_{c}$ of every soil, regardless of USCS class, because values for $C_{u}$ and $C_{c}$ can indicate soil grading and uniformity regardless of soil type. The USCS classification of a soil is largely included in the protocol as a qualitative parameter because, other than $C_{u}$ and $C_{c}$, the quantitative factors that determine the USCS class of a soil have little or no bearing on sensor response.

\subsubsection{Physical parameters}

Dry bulk density and porosity are commonly measured in laboratory studies of soils. Dry bulk density is defined as the mass of the mineral aggregates divided by the total volume they occupy in the soil. This parameter depends greatly on the mineral assemblage of the soil and on changes in the soil such as consolidation and cementation. Porosity is a measure of the void space that is available to hold water in a soil. Both dry bulk density and porosity affect the thermal properties of a soil (Howington et al. 2010; van Dam et al. 2005). These parameters are inversely related; the more voids in a soil, the lower the value of soil density and the higher its porosity (ASTM Standard D1895-96, 2010). This relationship is not linear and depends on many other factors including grain size, sorting, and particle density. Thus, both parameters are included in the protocol.

\subsubsection{Hydraulic parameters}

Effective saturation $\left(S_{e}\right)$ is a normalized, dimensionless measure of water content (van Genuchten 1980) derived from the water retention curve of a soil. The water retention curve represents the relationship between water content and water potential (i.e., ability of water to move in porous media) of soils and is characteristic of different soil types (Mualem 1976; Equation 3). The shape of the water retention curve can be defined using different models. One of the most commonly used models, the one used 
here, is the Mualem-van Genuchten model (Fredlund and Xing 1994; Mualem 1976; van Genuchten 1980).

$$
S_{e}=\frac{\left(\theta-\theta_{r}\right)}{\left(\theta_{s}-\theta_{r}\right)}
$$

where:

$$
\begin{aligned}
S_{e}= & \text { effective saturation; } \\
\theta= & \text { volumetric water content, the fraction of the total volume of } \\
& \text { soil occupied by the water contained in the soil; } \\
\theta_{r}= & \text { residual water content, the water content which requires a } \\
& \text { large suction change to remove additional water from the soil. } \\
\theta_{s}= & \text { saturated water content, the moisture content when all pore } \\
& \text { space is occupied by water. }
\end{aligned}
$$

The effective saturation of a soil can also be calculated using its hydraulic conductivity - a directly measured soil property. Ideally, only directly measured hydraulic parameters would be used in the protocol, and hydraulic conductivity would be used instead of effective saturation. Unfortunately, estimating the hydraulic conductivity of soil is difficult because it is highly variable among in-situ soils, collecting undisturbed samples is difficult, and measuring it in a laboratory requires significant resources. Because of these difficulties and because the water content of a soil is considered critical to sensor response (Chan and Knight 1999; Koh and Wakeley 2009; Robinson et al. 2003; Roth et al. 1992), effective saturation was used in the protocol. Parameters derived from water retention curves are also part of the standard of practice for hydrologic characterization of unsaturated soils (ASTM Standard D6836-02, 2008).

\subsubsection{Electrical parameters}

Specific conductance is a measure of the ability of a material to conduct an electric current and is the inverse of resistivity. Normally, resistivity is measured and then converted into specific conductance. Because many sensors use electromagnetic radiation to measure material properties (Jensen 2000) and soil conductivity will directly affect signal attenuation, specific conductance is important for characterizing sensor response to soils. 


\subsubsection{Thermal parameters}

Thermal resistivity is the inverse of thermal conductivity, which is the ability of a material to conduct heat. Thermal properties affect how the soil responds to heat and are important in the detection of buried objects and purposefully disturbed soils (Curtis 2001). Thermal diffusivity is a measure of how quickly the temperature of a material adjusts under changing conditions. It is equal to the thermal conductivity $(K)$ divided by the product of its density $(\rho)$ and specific heat capacity $(C)$, (Equation 4 ) also known as its volumetric heat capacity.

$$
a=\frac{K}{\rho C}
$$

where:

$$
\begin{aligned}
& \alpha=\text { thermal diffusivity } \\
& K=\text { thermal conductivity } \\
& C=\text { specific heat capacity } \\
& \rho=\text { density }
\end{aligned}
$$

\subsubsection{Geologic parameters}

The geologic parameters used in the protocol include quantitative mineralogy and field notes and descriptions. Because of their dependence on field conditions and sample quality, these parameters were available for only a limited number of sites. Mineralogical data were acquired using a PANalytical transmission X-ray diffractometer (XRD) running HighScore Plus (C) software. The software compares the ratio between integrated diffraction intensities of the samples to those of a cobalt standard to calculate mineral abundances. Mineral composition, particularly the abundance of silicates (e.g., quartz and feldspar), clay minerals, and some evaporates, such as gypsum, can greatly affect sensor response (Arcone et al. 2008; Howari et al. 2007) and were thus an integral part of the protocol development.

Field notes and descriptions were used to evaluate all other soil parameters in the protocol with the geologic setting of the sample sites. This component of the protocol is essential because of the distinct influence of geologic architecture on soil material composition. Field notes and descriptions also 
served as a developmental segue between the initial steps of the GEOTACS Program and the construction of full-scale, realistic 3-D geologic models.

\subsection{Descriptive statistics}

Most statistical tools are designed to find systematic patterns in a population or dataset. Their efficacy and use are also often highly dependent on the size and distribution of the dataset being studied. Descriptive statistics are used to describe the main features of a dataset quantitatively and to summarize other important points (e.g., type of distribution, covariance) qualitatively. Because descriptive statistics provide the background information needed to determine the usefulness of certain statistical tools, they must be performed before true analyses can be conducted. Thus, a number of methods were used to evaluate the general character of the soil properties datasets used in this study. They include probability and box plots; skewness, kurtosis, and normality tests; and calculations of mean and standard deviation.

\subsubsection{Soil parameter datasets}

The combined dataset used in this study is relatively large, having up to 243 data values per soil parameter. However, when divided into its 20 AOI sources, a single dataset for one soil property in one AOI generally has fewer than 15 values. Unfortunately, small datasets can sometimes inhibit robust statistical analysis of the data (StatSoft 2010). Moreover, the complexity of our data (i.e., 20 AOIs, up to 243 data points per parameter, and nine quantitative parameters) complicated the statistical approach that had to be used. Our approach had to cull statistically meaningful information, including quantitative comparisons between each data component, while adjusting for the difficulties of small data groups.

Most traditional statistical methods are parametric, meaning that they assume there is some predictable distribution to the data. More often than not, these methods require normally distributed data. It is also assumed that the true mean value of the dataset falls within the data range being analyzed (StatSoft 2010). These assumptions are reasonable for the largest of our datasets, but as detailed later in this chapter, descriptive statistics are unreasonable for our smallest dataset. Our data thus posed a unique problem; in one form they fit many traditional statistical methods, and in others they did not. The following sections describe our investigation of the factors influencing this discrepancy. 


\subsubsection{Types of descriptive statistics}

Descriptive statistics include a number of tests, simple calculations, and comparisons. They are used to understand the characteristics of the data being analyzed and help determine the types of further statistical analyses that should be used (Mathews 2005). Information about the distribution, central tendency, and dispersion of data can be obtained using descriptive statistics. Each set of information is comprised of several factors (e.g., mean, median, and quartile distribution). The factors used in this study were (1) mean, (2) maximum, (3) minimum, (4) standard deviation, (5) normality, (6) skewness, and (7) kurtosis. Besides simple calculations, three other tools were used: probability plots, box plots, and histograms.

Probability plots were made against a normal probability curve to test whether or not the datasets are normally distributed ${ }^{1}$. Box plots (also known as box-and-whisker diagrams) are a graphic way of depicting groups of numerical data through their five-number "summary" - the minimum observed value, the lower quartiles, the median quartiles, the upper quartiles, and the maximum observed value. Box plots are a visual way to show the outliers of a population (Massart et al. 2005). Histograms of each parameter were plotted to test for skewness and kurtosis. Skewness is a measure of asymmetry of the probability distribution of a variable (Davis 2002). It can be described as strongly positively or strongly negatively skewed and other variations, as shown in Table 2. A positive skewness means that the distribution has relatively few high values, whereas a negative skewness means that the distribution has relatively few low values.

Table 2. Skewness descriptive terminology (Prothero and Schwab, 1996).

\begin{tabular}{|l|l|}
\hline Skewness Value & Descriptive term \\
\hline$>0.30$ & Strongly positively skewed \\
\hline 0.30 to 0.10 & Positively skewed \\
\hline 0.10 to -0.10 & Near-symmetrical (unskewed) \\
\hline-0.10 to -0.30 & Negatively skewed \\
\hline$<-0.30$ & Strongly negatively skewed \\
\hline
\end{tabular}

Kurtosis measures the "peakedness" of the probability distribution of a variable (Davis 2002). It can be described as leptokurtic, mesokurtic, or

\footnotetext{
${ }^{1}$ Normality was also tested using the Anderson-Darling normality test (Mathews 2005).
} 
platykurtic (Table 3). A higher kurtosis means more of the variance ${ }^{1}$ in a dataset is the result of infrequent extreme deviations ${ }^{2}$, as opposed to frequent modestly sized deviations.

Table 3. Kurtosis descriptive terminology(Prothero and Schwab, 1996).

\begin{tabular}{|l|l|}
\hline Kurtosis Value & Descriptive term \\
\hline$>1.0$ & Excessively peaked (leptokurtic) \\
\hline 1.0 & Normally peaked (mesokurtic) \\
\hline$<1.0$ & Deficiently peaked (platykurtic) \\
\hline
\end{tabular}

\subsubsection{Applications of descriptive statistics}

The descriptive statistics introduced in the previous section were performed for eight quantitative parameters using data from 13 sites. Mineralogy was not included because of the small size of the dataset. A summary of the soil parameters and the corresponding descriptive statistics is shown in Table 4. When data for all 13 sites are considered as a single population, the smallest number of values in a single data group is 44 (for specific conductance). Porosity, dry bulk density, and effective saturation have the maximum number, which is 243 (Table 4). Probability plots for each parameter (Figure 2) reveal that none of the soil parameters have a normal distribution. Summaries of each parameter's descriptive statistics, including histogram and box plot, are shown in Figures 3 through 10. These figures also reveal non-normal distributions for all parameters.

Table 4. Summary for descriptive statistics of quantitative parameters. $C_{u}=$ coefficient of uniformity; $C_{c}=$ coefficient of curvature; Se = effective saturation; $\varphi=$ porosity; $\rho=$ dry bulk density; $\sigma=$ specific conductance; $R=$ thermal resistivity; $\alpha=$ thermal diffusivity.

\begin{tabular}{|l|l|r|r|r|l|l|l|}
\hline Parameter & N & Mean & $\begin{array}{l}\text { Standard } \\
\text { Deviation }\end{array}$ & $\begin{array}{l}\text { Maximum } \\
\text { Value }\end{array}$ & $\begin{array}{l}\text { Minimum } \\
\text { Value }\end{array}$ & Skewness & Kurtosis \\
\hline$C_{u}$ & 101 & 114.07 & \multicolumn{1}{|c|}{237.04} & 2000.00 & \multicolumn{1}{l}{2.25} & 5.64 & 40.88 \\
\hline$C_{c}$ & 101 & 4.27 & 9.92 & 67.24 & 0.613 & 4.56 & 23.09 \\
\hline$S_{e}$ & 243 & 0.41 & 0.31 & 0.99 & -0.03 & 0.41 & -1.13 \\
\hline$\varphi$ & 243 & 41.37 & 8.34 & 78.67 & 25.43 & 1.29 & 3.49 \\
\hline$\rho$ & 243 & 1.57 & 0.21 & 1.98 & 0.64 & -1.10 & 3.01 \\
\hline$\sigma$ & 44 & 422.23 & 570.06 & 2500.00 & 18.00 & 2.28 & 4.89 \\
\hline$R$ & 235 & 1.42 & 1.01 & 4.87 & 0.30 & 1.41 & 1.36 \\
\hline$\alpha$ & 235 & 0.54 & 0.41 & 2.15 & 0.12 & 1.92 & 3.43 \\
\hline
\end{tabular}

1 Variance denotes how far a set of numbers is spread out from each other (Davis 2002).

2 Deviation is a measure of the difference between an observed value and the mean (Davis 2002). 


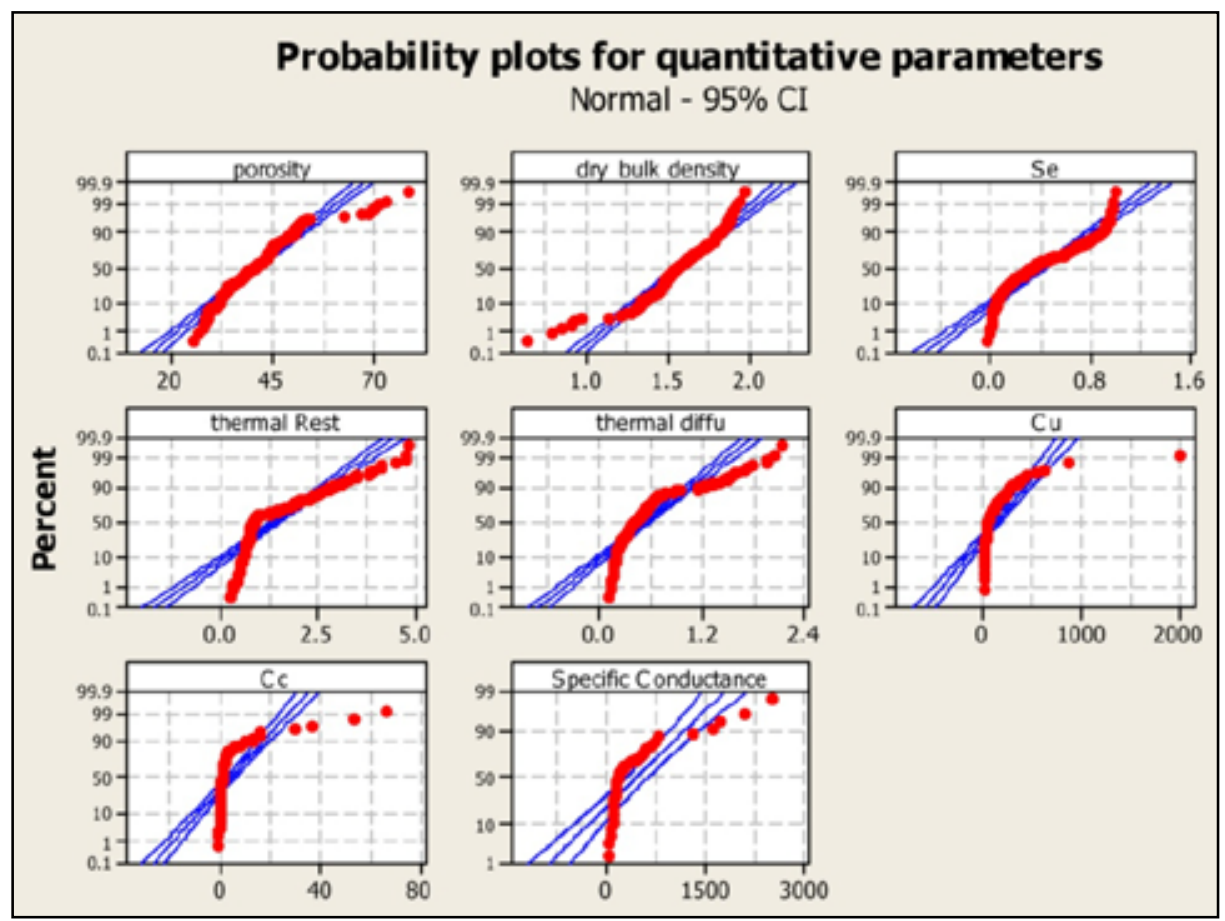

Figure 2. Probability plots for quantitative parameters. Blue lines represent the 95 percent confidence interval for a normal distribution. As shown by the data values for the parameters (red dots), all parameters have a non-normal distribution.

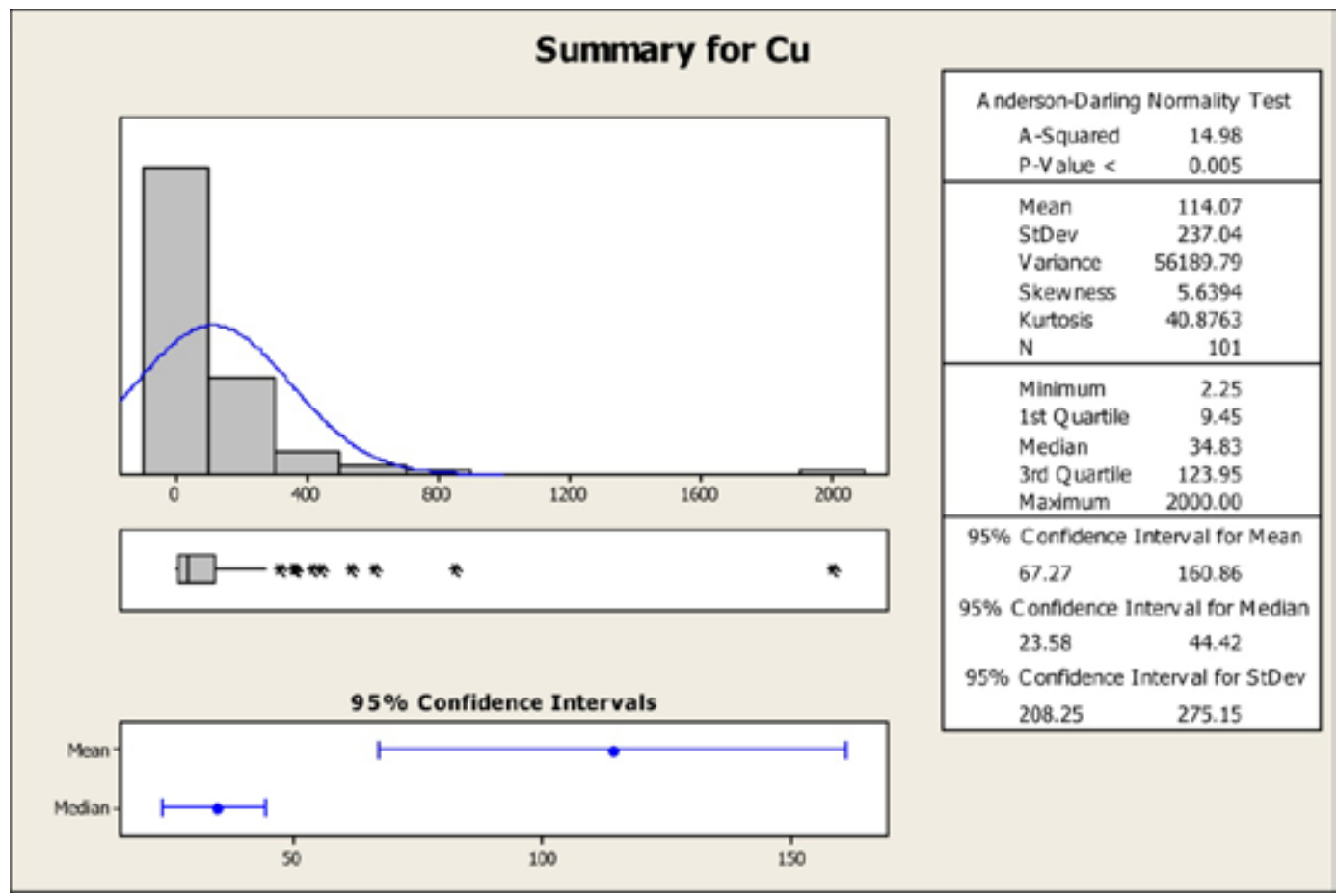

Figure 3. Summary statistics for the coefficient of uniformity (Cu). The histogram on top shows the data being strongly negatively-skewed. Histogram is leptokurtic. The corresponding box plot and 95 percent confidence intervals for mean and median are below histogram. 


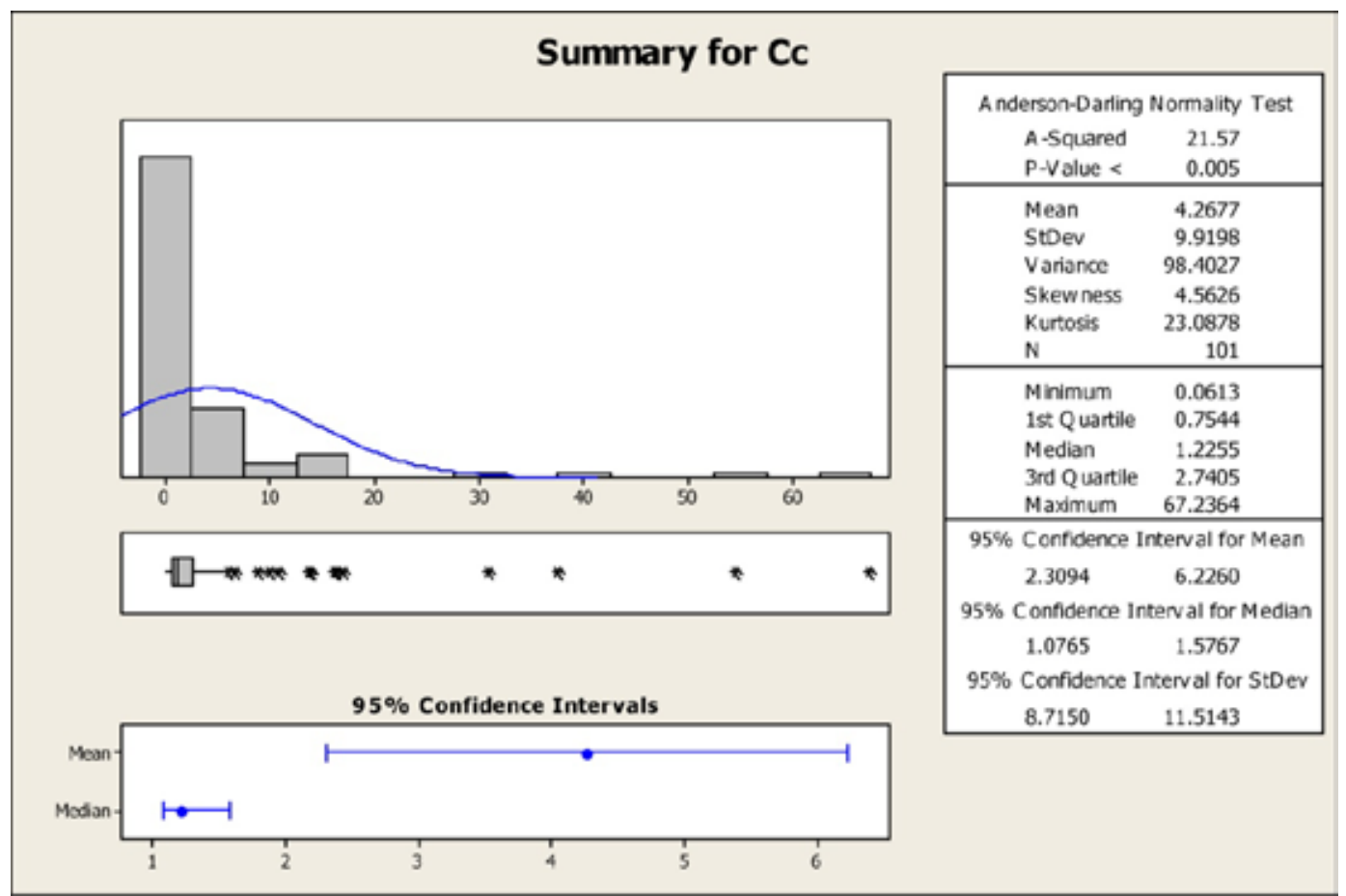

Figure 4. Summary statistics for the coefficient of curvature (Cc). The histogram on top shows the data being strongly negatively-skewed. Histogram is leptokurtic. The corresponding box plot and 95 percent confidence intervals for mean and median are below histogram.

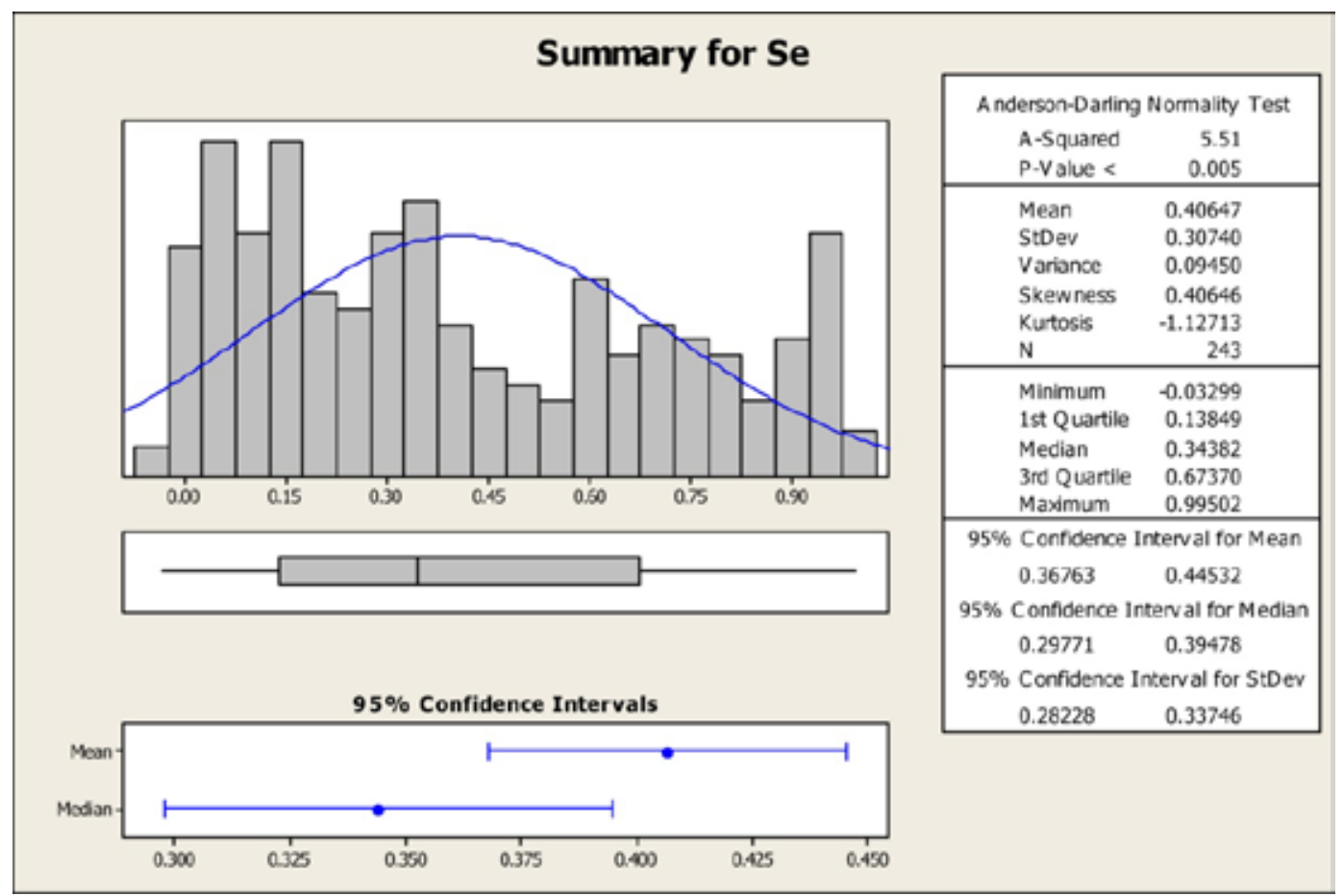

Figure 5. Summary statistics for the effective saturation (Se). The histogram on top shows the data being negatively-skewed. Histogram is platykurtic. The corresponding box plot and 95 percent confidence intervals for mean and median are below histogram. 


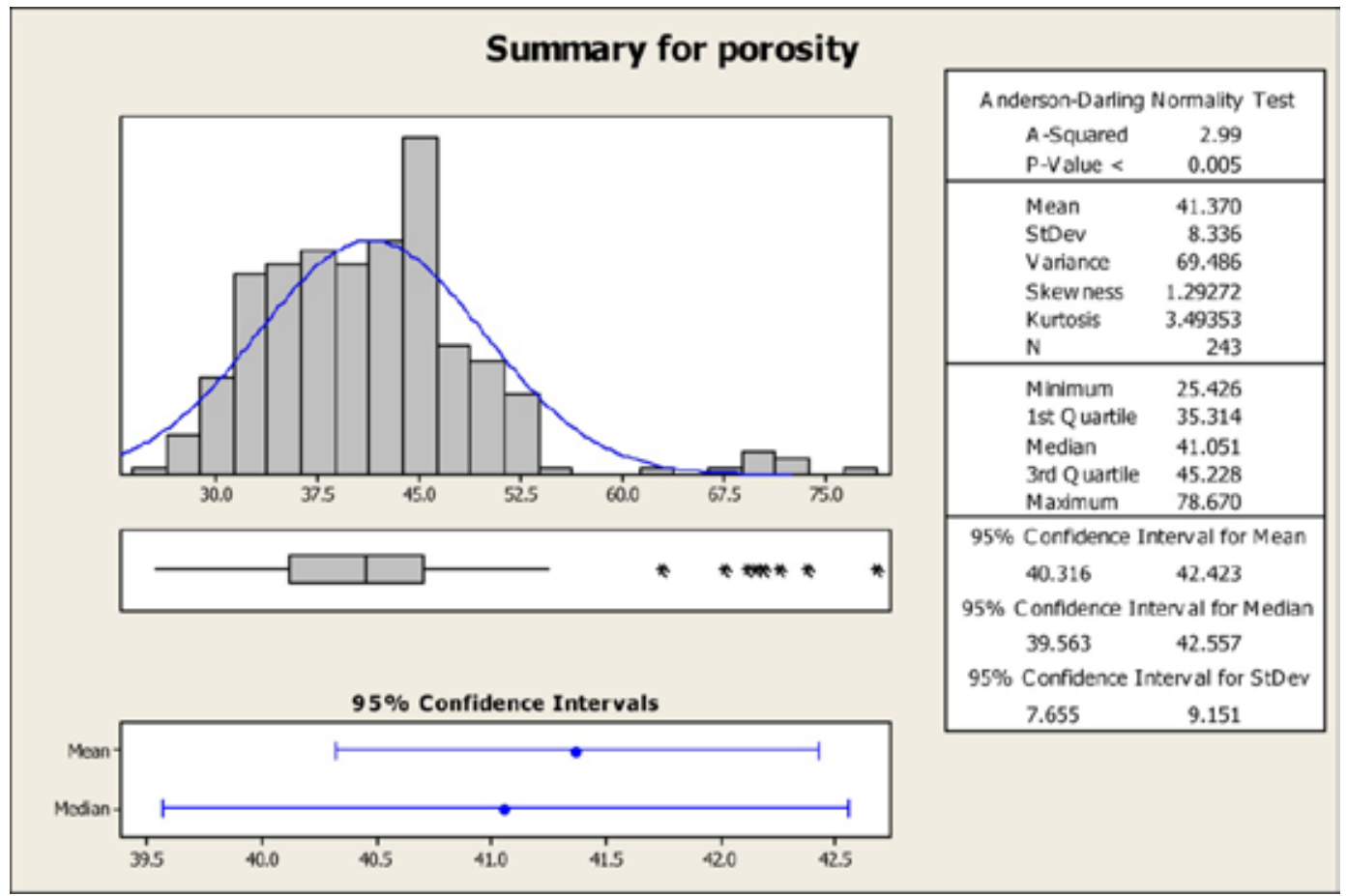

Figure 6. Summary statistics for porosity $(\varphi)$. The histogram on top shows the data being strongly negatively-skewed. Histogram is leptokurtic. The corresponding box plot and 95 percent confidence intervals for mean and median are below histogram.

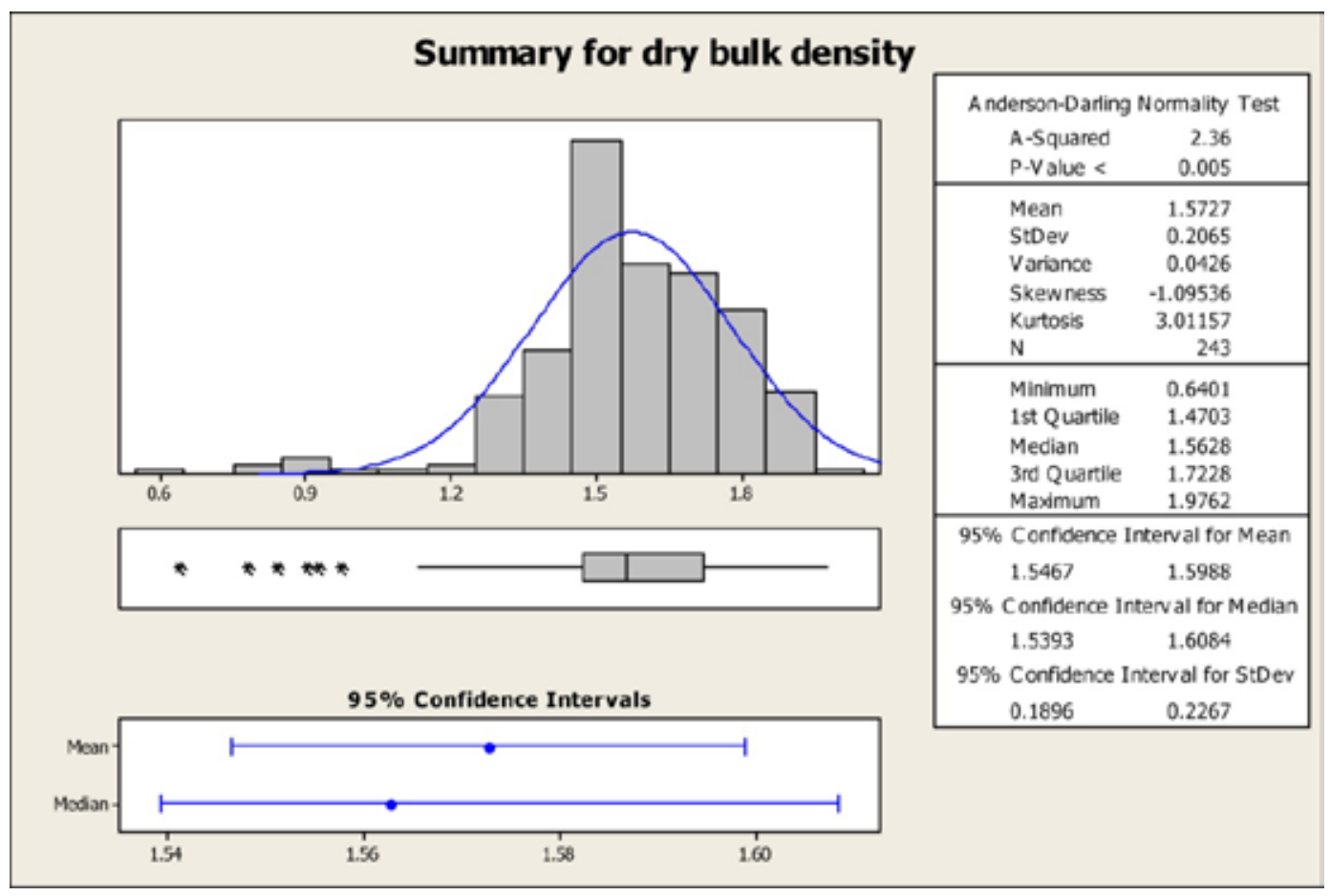

Figure 7. Summary statistics dry bulk density ( $\rho)$. The histogram on top shows the data being strongly positively-skewed. Histogram is leptokurtic. The corresponding box plot and 95 percent confidence intervals for mean and median are below histogram. 


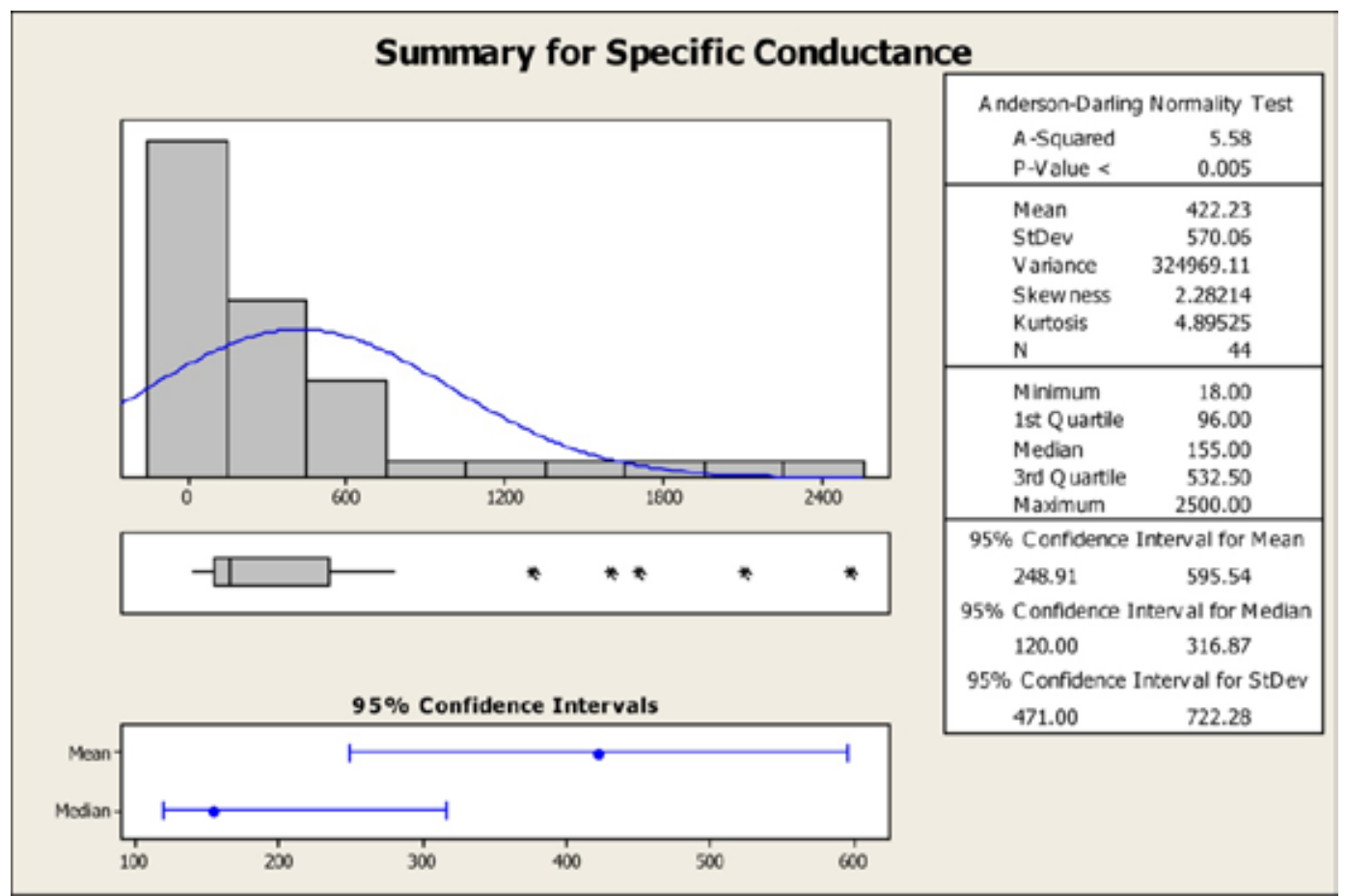

Figure 8. Summary statistics for specific conductance $(\sigma)$. The histogram on top shows the data being strongly negatively-skewed. Histogram is leptokurtic. The corresponding box plot and 95 percent confidence intervals for mean and median are below histogram.

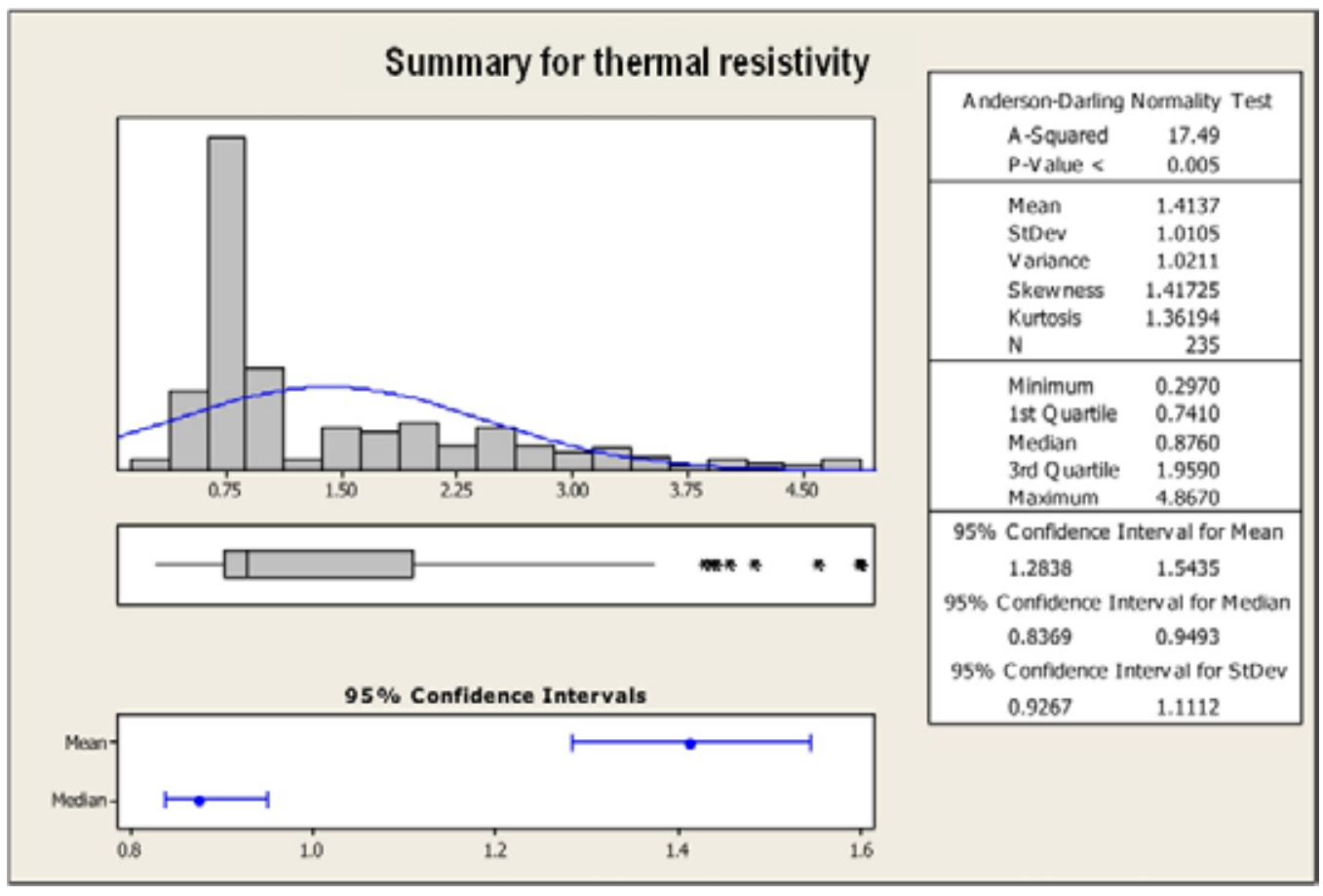

Figure 9. Summary statistics for thermal resistivity (R). The histogram on top shows the data being strongly negatively-skewed. Histogram is leptokurtic. The corresponding box plot and 95 percent confidence intervals for mean and median are below histogram. 


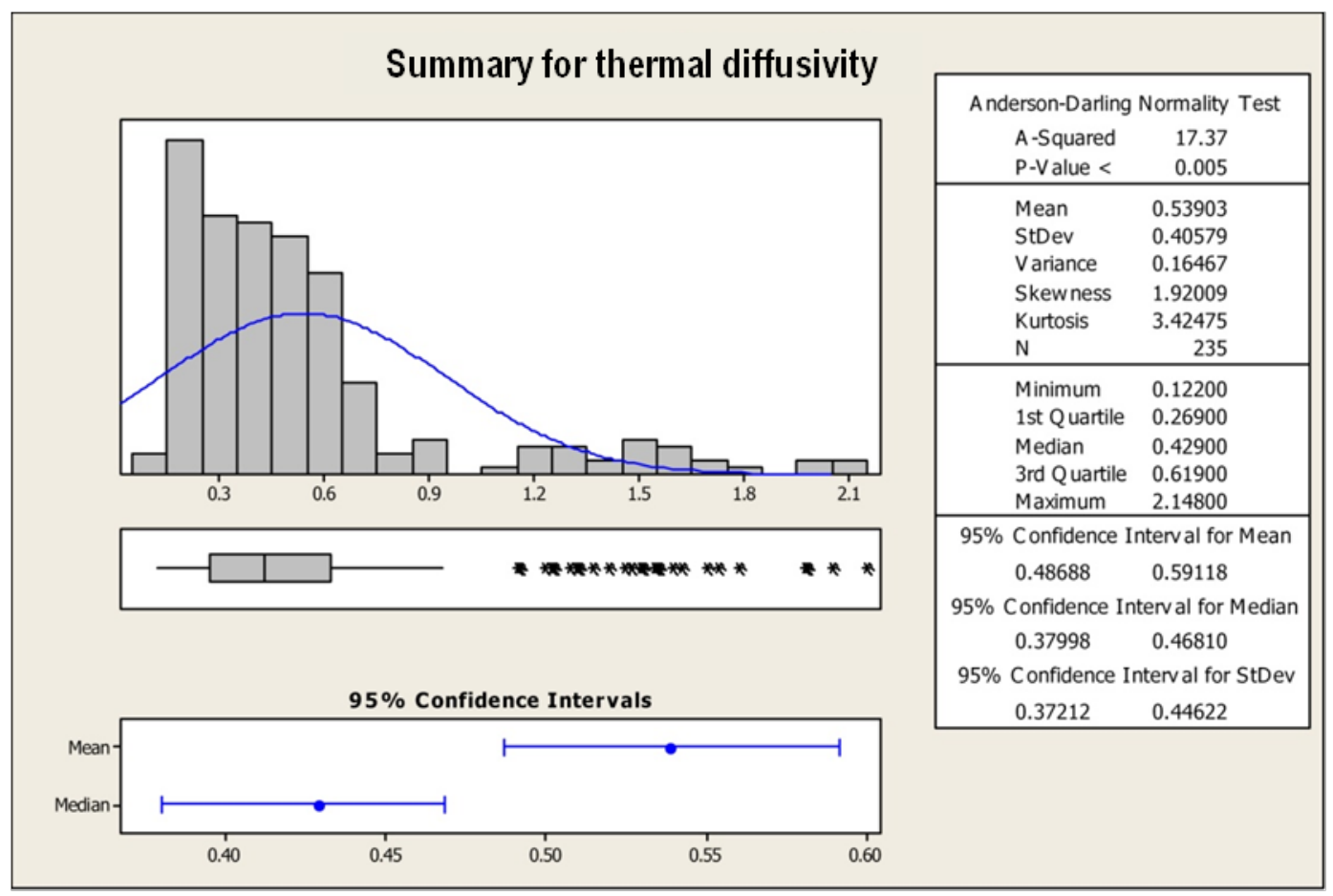

Figure 10. Summary statistics for thermal diffusivity $(\alpha)$. The histogram on top shows the data being strongly Inegatively-skewed. Histogram is leptokurtic. The corresponding box plot and 95 percent confidence intervals for mean and median are below histogram.

Coefficient of uniformity, coefficient of curvature, effective saturation, porosity, density, specific conductance, resistivity, and diffusivity are strongly positively skewed (their skewness values are >0.30; Figures $3-6$ and Figures 8 -10). On the other hand, dry bulk density is strongly negatively skewed (skewness value <-0.30; Figure 7). All parameters except specific conductance follow a leptokurtic pattern (excessively peaked histogram; Figures 3 and 4 and Figures 6 - 10), whereas effective saturation follows a platykurtic pattern (deficiently peaked histogram; Figure 5).

\subsubsection{Summary}

The available soils data were heavily skewed, exhibited strong kurtosis, and were non-normally distributed. Their means fell within the data ranges, but they contained a large number of outliers, which contributed to the skewness and kurtosis of the datasets. In statistical analysis, nonnormal multivariate data (like those in question) are often analyzed with non-parametric methods. This is because they do not conform to the rules and assumptions of parametric methods and, as a result, may not yield statistically significant results. It would seem, then, that the obvious choice for our inductive analysis would be a non-parametric method. 
Non-parametric methods have the distinct advantage over parametric methods in that they rely on fewer assumptions. Thus, in most cases, they are more robust. But because they have less power (i.e., lower probability that the result will reject the null hypothesis), non-parametric methods usually require larger datasets to yield statistically significant results (i.e., P-Value less than 0.005).

The soils-properties dataset available for this study did not satisfy all of the assumptions of parametric methods. However, it was a relatively small dataset, and research aims required that we compare even smaller groups of data within the larger dataset ( $\leq 15$ data points) and evaluate the differences among those groups (20 groups). At this scale of comparison, non-parametric tests do not provide statistically significant results. Yet our challenge was to develop a robust statistics-based method that could be applied at this scale.

\subsection{Inductive analysis of soil parameters}

Our primary task was to create a reasonable, reproducible, quantitative method to classify soil groups based on a set of soil properties. Because both parametric and non-parametric statistical approaches were inadequate, we decided to test the efficacy of standardizing the data. We chose the standard score, or Z-score, method (Jackson 2009). This method is well known, commonly used, and relatively simple to implement. After undergoing the Z-score method, soil materials were delineated via qualitative soil parameters and a "majority rule" in which the classification of a certain set of data points was based on the majority of the parameters. That is, if the majority of parameters indicated the series of corresponding data values was the same material, then the entire set was classified as that material.

\subsubsection{Normalization-based analysis}

All soil parameters were normalized using the Z-score normalization method. This method illustrates the relative difference between a given data value in a range and the mean of that range. The $Z$-score of a value (Equation 5) is the number of standard deviations that the value falls above or below the mean of all data values in the range. Each data value can then be compared to the range of values (and other single values) by subtracting the relative magnitudes of the Z-scores for each data value. 


$$
z=\frac{x \mu}{\sigma}
$$

where:

$$
\begin{aligned}
z & =\mathrm{Z} \text {-score value; } \\
\mu & =\text { mean of the range } \\
x & =\text { data value } \\
\sigma & =\text { standard deviation. }
\end{aligned}
$$

Table 5 shows the dry bulk density values for different depth intervals and the normalized values for a site in Afghanistan. As shown, the Z-score method simplifies visual and mathematical comparisons of nearly identical data values. Note that the "sets" of data to which the majority rule applies are the depth ranges; for every site, the array of soil properties was measured at several depth intervals.

Table 5. Example of using Z-score normalization to reveal relationships between data values at depth. Colors represent individual material types. Standard values at depths 100 to $140 \mathrm{~cm}$ (relative to all depths) showed a difference of $<0.50$; thus, they were grouped into one material type based only on the dry-bulk-density parameter. Density values were missing in the upper $60 \mathrm{~cm}$ at this site.

\begin{tabular}{|c|l|l|}
\hline Sample & $\boldsymbol{\rho}$ & $\boldsymbol{\rho}$ std \\
\hline $0-20$ & N/A & N/A \\
\hline $20-40$ & N/A & N/A \\
\hline $40-60$ & N/A & N/A \\
\hline $60-80$ & 1.530 & 1.440 \\
\hline $80-100$ & 1.515 & 0.470 \\
\hline $100-120$ & 1.502 & -0.390 \\
\hline $120-140$ & 1.504 & -0.31 \\
\hline $140-160$ & 1.490 & -1.210 \\
\hline
\end{tabular}

\subsubsection{Comparisons of normalized values}

The difference between the Z-score values is used to determine soil materials. But in order to assign that material type, some magnitude-ofdifference threshold must be used to compare the data. Because the threshold is an increment of Z-score ranges, it is also an increment of the soil-parameter ranges. There is a minimum difference in soils' properties a given sensor can detect (i.e., sensor fidelity), and the threshold determines 
the minimum parameter difference between any two materials. Thus, the threshold should be calculated using the fidelity of the sensor.

The number of soil materials that can be determined by a sensor in a given AOI depends on environmental conditions and sensor fidelity. Hence, rather than setting the magnitude-of-difference threshold to a value calculated for a certain scenario, we chose to use a process of trial and error to determine the maximum value that would yield a many-to-one relationship between the number of depth ranges and materials for each site. That is, we tested several thresholds until we discovered a value that would produce a result that showed the same material at two or more depths at every site. The goal was to prove (1) that it is possible to classify soil materials at depth in multiple locations and (2) that the adjustment of the threshold value will be versatile enough to capture variability in site conditions and sensor fidelities. After several iterations, a value of 0.50 was chosen.

When the absolute difference of Z-scores (Equation 6) for a single parameter at any two depth ranges was less than 0.50, those depths were grouped as one material. This was done for each soil parameter in the protocol. Each parameter's set of material types was then compared with the others. The final set of materials was determined by selecting the material type that occurred most frequently across all parameters.

$$
Z_{1}-Z_{2}=|Z|
$$

where:

$Z_{1}=$ the Z-score value of depth range 1 ;

$Z_{2}=$ the Z-score value of depth range 2;

$|Z|=$ the absolute difference of the Z-score.

\subsection{Application of the protocol}

To test the protocol, we applied it to a subset of our dataset. Data from four AOIs--one in Iraq, one in the U.S., and two in Afghanistan--were analyzed using the methods described in previous sections. All data were divided into samples, which corresponded to depth intervals. Only the Iraq site had data for quantitative mineralogy. The U.S. site had the largest number of samples: the Afghanistan site, the fewest. The sites were chosen from the larger group because of their data quality and their relevance to GEOTACS Program needs. 


\subsubsection{Site B, Iraq}

Twelve samples were available at depth intervals of 10 to $20 \mathrm{~cm}$ to a total depth of $1.9 \mathrm{~m}$. Data for all the parameters at all depth ranges were available, with the exception of mineralogy at the 110-cm depth. Raw parameter values (i.e., non-normalized data) are shown in Appendix A. Standardized values are shown in Table 6. Final material definition for the site is shown in Table 7. According to the USCS soil classification, the upper $50 \mathrm{~cm}$ of the subsurface is silty sand. From 70 to $90 \mathrm{~cm}$ in depth, the soil is classified as well-graded sand with silt and gravel. Below the 100-cm depth, the soil is poorly-graded sand. Using our threshold of 0.50 for the absolute difference resulted in 10 material types out of 12 samples (Table 7).

Of all the quantitative parameters, specific conductance showed the most uniformity (using the 0.50 threshold); five adjacent depth ranges (10 to $90 \mathrm{~cm}$ ) were classified as the same material. The least uniform property for the CBU site was content of the mineral albite, showing similar data values and materials at only two depths: 150 and $170 \mathrm{~cm}$.

\subsubsection{Site CUL2, U.S.}

Twenty-one samples were available at depth intervals of $10 \mathrm{~cm}$ to a total depth of $2 \mathrm{~m}$. Mineralogy and specific conductance data were not available for this site. USCS, $\mathrm{C}_{\mathrm{u}}$, and $\mathrm{C}_{\mathrm{c}}$ data were available for alternating depth

Table 6. Standardized values for CBU site. Colors by column represent groups where there is an absolute $Z$-score difference of less than 0.50 . Sample= sampling ID and depth in $\mathrm{cm}$; Std=standardized value; USCS= Unified Soil Classification System; $\mathrm{C}_{\mathrm{u}}=$ coefficient of uniformity; $\mathrm{C}_{\mathrm{c}}=$ coefficient of curvature; $\mathrm{Se}=$ effective saturation; $\varphi=$ porosity; $\rho=$ dry bulk density; $\sigma=$ specific conductance; $R=$ thermal resistivity; $\alpha=$ thermal diffusivity;

$\mathrm{Qtz}=$ quartz; Alb=albite; Ksp=Potassium-rich feldspars; Clay=total clay mineral content; Carb=carbonate.

\begin{tabular}{|c|c|c|c|c|c|c|c|c|c|c|c|c|c|}
\hline Sample & ASTM Classification & std Cu & $\operatorname{std} C_{c}$ & $\phi$ std & $\rho$ std & $\sigma$ std & R std & $\alpha$ std & Qtz std & Alb std & Ksp std & Clay stod & darb stc \\
\hline site $B$ 0-1,2 & Silty sand (SM) & -1.21 & -1.178 & 0.21 & -0.02 & 0.023 & 2.292 & -1.5 & -0.268 & -0.373 & -1.815 & 2.233 & 0.914 \\
\hline site B -10-1,2 & Silty sand (SM) & 0.255 & 1.273 & 0.51 & $\mid-0.407$ & $\mid-0.613$ & $0.867 \mid$ & -0.86 & $-0.071 \mid$ & -1.684 & -1.054 & -1.318 & 1.764 \\
\hline site B-31-1,2 & Silty sand (SM) & 1.092 & 1.429 & 0.95 & $-0.857 \mid$ & $\mid-0.431$ & -0.747 & 0.49 & -1.447 & 1.267 & $|-1.054|$ & -0.134 & 1.480 \\
\hline site $B-50-1,3$ & Silty sand (SM) & 1.878 & 0.136 & 1.3 & -1.384 & $\mid-0.841$ & $\mid-1.619$ & 1.44 & -0.071 & -0.700 & 1.227 & 0.457 & 0.064 \\
\hline site $B-70-1,3$ & Well-graded sand with silt and gravel (SW-SM)g & 1.171 & 0.459 & -0.09 & -0.169 & -0.804 & -0.355 & 0.71 & -0.563 & 0.119 & -0.104 & 0.161 & 0.348 \\
\hline site $B-90-1,2$ & Well-graded sand with silt and gravel (SW-SM)g & 0.512 & 1.725 & 1.49 & -1.491 & -0.841 & 0.237 & 0.75 & 0.125 & -0.045 & -0.104 & 0.901 & -0.502 \\
\hline site $B-100-2,3$ & Poorly-graded sand (SP) & -0.187 & -1.042 & -0.96 & 0.898 & -0.158 & -0.019 & -0.43 & -0.366 & 1.594 & -0.104 & 0.309 & -0.927 \\
\hline site B-110-1,2 & Poorly-graded sand (SP) & -0.478 & -0.633 & -1 & 0.939 & -0.613 & -0.098 & -0.36 & N/A & $\mathrm{N} / \mathrm{A}$ & $\mathrm{N} / \mathrm{A}$ & $\mathrm{N} / \mathrm{A}$ & $\mathrm{N} / \mathrm{A}$ \\
\hline site B-130-2,3 & Poorly-graded sand (SP) & -0.911 & -0.74 & -1.31 & 1.436 & -0.067 & $\mid 0.866$ & -1.22 & 1.304 & -0.700 & 0.847 & -0.134 & -0.785 \\
\hline site $B-150-1,3$ & Poorly-graded sand (SP) & -0.725 & -0.495 & -1.03 & 0.966 & 2.297 & -0.132 & -0.71 & 1.893 & -0.373 & 0.086 & -1.022 & -0.785 \\
\hline site $B-170-2,3$ & Poorly-graded sand (SP) & -0.187 & -0.59 & 0.75 & -0.745 & 0.478 & -0.552 & 1.51 & 0.714 & -0.373 & 0.847 & -0.726 & -0.502 \\
\hline site B-190-1,2 & Poorly-graded sand (SP) & -1.21 & -0.344 & -0.81 & 0.834 & 1.57 & -0.741 & 0.17 & -1.250 & 1.267 & 1.227 & -0.726 & -1.068 \\
\hline
\end{tabular}


Table 7. Final definition of material types for the Iraq site.

\begin{tabular}{|l|l|}
\hline Depth $(\mathbf{c m})$ & Material Type \\
\hline 0 & 1 \\
\hline 10 & 2 \\
\hline 30 & 3 \\
\hline 50 & 4 \\
\hline 70 & 5 \\
\hline 90 & 6 \\
\hline 100 & 7 \\
\hline 110 & 7 \\
\hline 130 & 8 \\
\hline 150 & 8 \\
\hline 170 & 9 \\
\hline 190 & 10 \\
\hline
\end{tabular}

intervals, as shown in Table 8. This site had the highest sampling frequency of any used in this study. Raw parameter values (i.e., non-normalized data) are shown in Appendix A. Standardized values are shown in Table 8. Final material definition is shown in Table 9.

Table 8. Standardized values for U.S. site. Colors by column represent groups where there is an absolute Z-score difference of less than 0.50. Sample= sampling ID and depth in $\mathrm{cm}$;

Std=standardized value; USCS= Unified Soil Classification System; $\mathrm{C}_{\mathrm{u}}=$ coefficient of uniformity; $\mathrm{C}_{c}=$ coefficient of curvature; $\mathrm{Se}=$ effective saturation; $\varphi=$ porosity; $\rho=$ dry bulk density; $\mathrm{R}=$ thermal resistivity; $\alpha=$ thermal diffusivity.

\begin{tabular}{|c|c|c|c|c|c|c|c|c|}
\hline Sample & uscs & std Cu & std Ce & Se std & Qstal & psta & R std & asta \\
\hline CULZ-O & Silty sand (SM) & 1.792 & 1.533 & 0.557 & 2.052 & 2.077 & $-2: 385$ & 3.856 \\
\hline CUL2-10 & - & - & - & 0.47 & -1.436 & 1,488 & $-1,603$ & 1.057 \\
\hline CUL2-30 & Silty sand (SM) & 0.928 & 2,009 & -1.334 & 0.971 & 0.927 & 0.065 & 0.658 \\
\hline CUL2-4O & - & - & - & 0.456 & 0.183 & 0.25 & 0.086 & -0.326 \\
\hline CULZ-50 & Sandy silty clays (CL-ML) & 0,048 & 0.647 & 0.289 & -0.93 & 0.97 & 0.946 & 0.17 \\
\hline CULZ-70 & Sandy tean clays $(\mathrm{CL})$ & 1.427 & -0.663 & 0.352 & 0.34 & 0,293 & 2.004 & 0.659 \\
\hline CUL2-80 & - & - & - & -1.25 & -1.036 & 1.019 & 0.622 & 0.299 \\
\hline CUL2-90 & Silty sand (SM) & 0,457 & 0.475 & -2.707 & -0.311 & 0.258 & 0,787 & -0.14 \\
\hline CULZ-100 & - & - & - & -1.327 & 0.154 & -0.219 & 0.721 & 0.167 \\
\hline CULZ-110 & Lean clay (CL) & 0.619 & 0.562 & 0,374 & 0.788 & -0.631 & 0.127 & -0.519 \\
\hline CULZ-160 & - & - & - & 0.752 & 0.265 & -0.335 & -0.098 & 0.52 \\
\hline CUL2-170 & Lean clay (CL) & 0.792 & 0.636 & 0.9 & 1.061 & -0.844 & 0.156 & -0.552 \\
\hline CULZ-180 & - & - & - & 0.715 & 1.076 & $-1,179$ & 0.3 & -0.798 \\
\hline CUL2-190 & Silt (ML) & 0.757 & 0.566 & 0.905 & 1.353 & -1.398 & 1.628 & 0.763 \\
\hline CULZ-200 & - & - & - & 0.874 & 0.951 & -1.048 & 0.721 & 0.434 \\
\hline
\end{tabular}


Table 9. Final definition of material types for the U.S. site.

\begin{tabular}{|l|l|}
\hline Depth $(\mathbf{c m})$ & Material Type \\
\hline 0 & 1 \\
\hline 10 & 2 \\
\hline 20 & 2 \\
\hline 30 & 3 \\
\hline 40 & 3 \\
\hline 50 & 4 \\
\hline 60 & 4 \\
\hline 70 & 5 \\
\hline 80 & 6 \\
\hline 90 & 7 \\
\hline 100 & 7 \\
\hline 110 & 8 \\
\hline 120 & 8 \\
\hline 130 & 9 \\
\hline 140 & 9 \\
\hline 150 & 10 \\
\hline 160 & 10 \\
\hline 170 & 10 \\
\hline 180 & 10 \\
\hline 190 & 10 \\
\hline 200 & 10 \\
\hline & \\
\hline
\end{tabular}

USCS soil classification for this site identifies silty sand at the surface and at the 30- and 90-cm depths. Sandy silty clay is identified only at $50-\mathrm{cm}$ depth. At $70 \mathrm{~cm}$, sandy lean clay is present. Silt occurs at 130 and $190 \mathrm{~cm}$. Lean clay occurs at 110-, 150-, and 170-cm depths. The protocol identified 10 material types at the site (Table 9). The coefficient of uniformity had the most similar values of all parameters, whereas thermal resistivity had the least.

\subsubsection{Site GA1 (Afghanistan)}

Eight samples were available at depth intervals of $20 \mathrm{~cm}$ to a total depth of $1.4 \mathrm{~m}$. Mineralogy and specific conductance data were not available for this site. Effective saturation, porosity, density, thermal resistivity, and thermal diffusivity data were not available from $\mathrm{o}-$ to $40-\mathrm{cm}$ depth. This site and GQ1 had the lowest sampling frequency of any used in this study. Raw parameter values (i.e., non-normalized data) are shown in Appendix A. 
Standardized values are shown in Table 10. Final material definition is shown in Table 11.

USCS soil classification for this site identifies silty gravel with sand at the surface and $20-\mathrm{cm}$ depth. Silty sand with gravel is identified at $40-\mathrm{cm}$ depth. Silty sand is identified at 6o-cm depth. Well-graded sand is identified at $80-\mathrm{cm}$ depth. Poorly-graded sand with silt is identified from 100 to $140 \mathrm{~cm}$. The protocol identified seven material types at the site (Table 11). The coefficient of uniformity had the most similar values of all parameters. Standard scores for the coefficient of curvature and porosity yielded identical material distributions.

Table 10. Standardized values for GA1 site. Colors by column represent groups where there is an absolute Z-score difference of less than 0.50. Sample= sampling ID and depth in $\mathrm{cm}$; Std=standardized value; USCS= Unified Soil Classification System; $\mathrm{C}_{\mathrm{u}}=$ coefficient of uniformity; $C_{c}=$ coefficient of curvature; Se=effective saturation; $\varphi=$ porosity; $\rho=$ dry bulk density; $R=$ thermal resistivity; $\alpha=$ thermal diffusivity.

\begin{tabular}{|c|c|c|c|c|c|c|c|c|}
\hline Sample & USCS & std Cu & stdCc & Se std & $\phi$ std & $\rho$ std & Rstd & $\alpha$ std \\
\hline GA1-0-20 & Silty gravel with sand (GM)s & 2.359 & -0.779 & - & - & - & . & - \\
\hline GA1-20-40 & Silty gravel with sand $(\mathrm{GM}) \mathrm{s}$ & 0.400 & -0.919 & - & - & _. & - & - \\
\hline GA1-40-60 & Silty sand with gravel(SM)g & -0.402 & 0.233 & - & - & 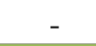 & - & 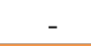 \\
\hline GA1-60-80 & Silty sand (SM) & -0.429 & 2.087 & -1.226 & -0.736 & 1.440 & 1.680 & -1.399 \\
\hline GA1-80-100 & Well-graded sand with silt (SW-SM) & -0.469 & -0.443 & -0.468 & -0.750 & 0.470 & -0.070 & -0.624 \\
\hline GA1-100-120 & Poorly-graded sand with silt (SP-SM) & -0.487 & -0.335 & -0.040 & -0.612 & -0.390 & -0.670 & 0.525 \\
\hline GA1-120-140 & Poorly-graded sand with silt (SP-SM) & -0.487 & 0.718 & 0.244 & 1.448 & -0.310 & -0.093 & 0.391 \\
\hline GA1-140-160 & Poorly-graded sand with silt (SP-SM) & -0.485 & -0.562 & 1.489 & 0.651 & -1.210 & -0.846 & 1.107 \\
\hline
\end{tabular}

Table 11. Final definition of material types for site GA1.

\begin{tabular}{|l|l|}
\hline Depth $(\mathrm{cm})$ & Material Type \\
\hline 0 & 1 \\
\hline 20 & 2 \\
\hline 40 & 3 \\
\hline 60 & 4 \\
\hline 80 & 5 \\
\hline 100 & 6 \\
\hline 120 & 6 \\
\hline 140 & 7 \\
\hline
\end{tabular}




\subsubsection{Site GQ1 (Afghanistan)}

Eight samples were available at depth intervals of $20 \mathrm{~cm}$ to a total depth of $1.4 \mathrm{~m}$. Mineralogy and specific conductance data were not available for this site. This site and GA1 had the lowest sampling frequency of any used in this study. Raw parameter values (i.e., non-normalized data) are shown in Appendix A. Standardized values are shown in Table 12. Final material definition is shown in Table 13.

Table 12. Standardized values for GQ1 site. Colors by column represent groups where there is an absolute Z-score difference of less than 0.50. Sample= sampling ID and depth in $\mathrm{cm}$; Std=standardized value; USCS $=$ Unified Soil Classification System; $\mathrm{C}_{\mathrm{u}}=$ coefficient of uniformity; $C_{c}=$ coefficient of curvature; $S e=$ effective saturation; $\varphi=$ porosity; $\rho=$ dry bulk density; $R=$ thermal resistivity; $\alpha=$ thermal diffusivity.

\begin{tabular}{|c|c|c|c|c|c|c|c|c|}
\hline Sample & USCS & std Cu & std Cc & Se std & $\phi$ std & $\rho$ std & R std & $\alpha$ std \\
\hline GQ1-2-D-0-1+2 & Sandy silt s(ML) & 1.189 & -0.57 & -1.09 & -2.26 & 2.278 & -0.62 & 0.812 \\
GQ1-2-D-20-1+2 & Silt with sand (ML)s & 0.298 & -0.41 & -0.89 & -0.16 & 0.087 & -0.04 & -0.37 \\
GQ1-2-D-40-1+2 & Silt (ML) & 0.304 & -0.37 & -0.68 & -0.03 & -0.13 & -1.86 & 1.888 \\
GQ1-2-D-60-1+2 & Silt (ML) & 0.127 & 0.623 & -0.79 & 0.127 & -0.29 & 0.309 & 0.189 \\
GQ1-2-D-80-1+2 & Silt (ML) & -0.56 & 0.038 & 0.199 & 0.948 & -1.12 & 1.002 & -0.13 \\
GQ1-2-D-100-1+2 & Silt (ML) & 1.272 & 2.2 & 0.745 & 0.044 & 0.03 & 1.24 & -0.12 \\
GQ1-2-D-120-1+2 & Silt (ML) & -1.29 & -0.77 & 0.954 & 0.495 & -0.23 & 0.493 & -1.07 \\
GQ1-2-D-140-1+2 & Silt (ML) & -1.34 & -0.74 & 1.556 & 0.831 & -0.63 & -0.52 & -1.19 \\
\hline
\end{tabular}

Table 13. Final definition of material types for site GQ1.

\begin{tabular}{|l|l|}
\hline Depth $(\mathrm{cm})$ & Material Type \\
\hline 0 & 1 \\
\hline 20 & 2 \\
\hline 40 & 2 \\
\hline 60 & 3 \\
\hline 80 & 4 \\
\hline 100 & 4 \\
\hline 120 & 5 \\
\hline 140 & 5 \\
\hline
\end{tabular}

USCS soil classification for this site identifies sandy silt at the surface. Silt with sand is identified at 20-cm depth. Silt is present from $40-$ to $140-\mathrm{cm}$ depth. The protocol identified six material types at the site (Table 13). Coefficient of curvature and the effective saturation had the most similar values of all parameters. Coefficient of uniformity, porosity, and density also showed a comparable distribution. 


\section{Discussion}

Previous methods used to define soil materials for 3-D models were founded on expert opinion with minimal statistical analyses of data (Wakeley et al. 2011). They relied primarily on qualitative judgments about geologic architecture: quantitative analyses of measured soil properties were used on a limited basis. The methods described in this report reverse that order of operations. That is, quantitative tools were developed to delineate soil materials based on measured properties data, and qualitative judgments about geologic conditions were used only to refine the material definitions. Both methods, however, recognize the importance of qualitative geologic judgments when defining soil materials for use in 3-D models for sensor response.

\subsection{Quantitative versus qualitative analysis}

Geologic environments of depositions were not included in the early method or in the protocol detailed here. Ideally, a depositional setting should be established for each site and soil material such that a relationship could be established between measured soil properties and site geomorphology. Unfortunately, multiple depositional settings can produce a single type of soil material. Likewise, a single geologic setting can produce multiple types of soil materials. Thus, direct correlations between geologic architecture and depositional environments are ambiguous because of their qualitative, interpretive nature.

Quantitative methods, on the other hand, may be able to distinguish among materials that appear to share a common geologic origin. While understanding the geologic setting of an AOI is critical for distributing materials in a 3-D model, that understanding is less critical for defining the soil materials themselves. When defining soil materials for sensor-response models, it is most important to use quantitative tools that compare sets of measured soil properties. Yet the relationships between soil properties can make the development of a quantitative process very complex.

Many soil properties have known relationships. For example, mineralogy can influence density, thermal resistivity, and specific conductance. These relationships can greatly affect sensor response (Koh and Wakeley 2010). They can also influence the results of statistical analyses if two or more 
properties have measured values that are some function of the other. Consider a clean, poorly-graded pure-quartz sand ${ }^{1}$ being analyzed using the method we developed here. Suppose that with a one percent volume increase of pure-quartz mineralogy, the specific conductance of the soil decreases by 1.5 percent, all other properties being equal. Without capturing this direct relationship in the quantitative analysis methods used to define soil materials for sensor simulations (or any other purpose), statistical methods may yield results that have reduced reliability. Nonetheless, as the applications and implementations of sensor systems proliferate, it is increasingly important to use quantitative analysis methods to classify soil materials.

\subsection{Limits and advantages of the new protocol}

The Z-score method introduced in this report is a new, unique application of statistical methods in support of 3-D subsurface modeling for sensor simulations. It allows the user to compare soil data that otherwise would be too similar to visually or qualitatively delineate soil materials (Table 5). The protocol also allows users to tailor each parameter to their research needs, making it applicable to numerous applications in subsurface modeling, sensor performance, and soil science. It provides numerous benefits over earlier methods used in the GEOTACS Program, including improved accuracy, more reproducible results, and increased reliability. Moreover, it removes some of the ambiguity associated with the expert judgment required to implement classification results in modeling efforts, which, in turn, makes the new protocol more consistent between geologic environments and more resistive to human error.

However, this version of the protocol has limitations, most of which hinge on a few factors. First, many soil properties are directly or indirectly correlated to one another (see the example in Section 5.1). In statistical terms, this problem is known as "co-variance." Co-variance can affect the weighting of the various parameters being analyzed and thereby alter the results. With respect to this protocol, co-variance may limit the user's ability to determine the materials that a sensor will "see." This is because the sensor is reacting to true ground conditions - all properties together, in-situ. The user of the protocol, on the other hand, is attempting to reconstruct what the sensor will "see" from non-in-situ data by making assumptions about the relationships between each measured soil property.

\footnotetext{
1 This type of material is extremely rare in nature and is used for explanatory purposes only.
} 
To define each material as it would be "seen" by the sensor, every correlation among all soil properties that impact sensor response would have to be characterized and documented carefully. This is a costly and time-consuming proposition for any project, and as such was an unrealistic goal for the GEOTACS Program. Thus, this limitation of such characterization was considered an acceptable margin of error in an effort to improve the state of practice for sensor simulations.

Second, the Z-score compares the deviation of a particular depth interval with the mean of all data points in the depth range. It does not compare the absolute deviation of a given interval from the data values measured in the intervals above and below it. That is, the protocol carries the assumption that the deviation of a single data point from the dataset's mean is more important than the deviation of two adjacent data points from each other. This assumption may not always hold true. Yet finding statistical methods to provide a better comparison between individual samples is difficult due to the nature of the data themselves: the datasets are very small, and the distribution is controlled by many geologic factors that cannot be "penciled in." Moreover, most of the traditional statistical methods are not meant to make comparisons between individual values. Most are intended to discover and analyze patterns in large datasets and are based on comparisons between a single data point and characteristics of the group (e.g., mean, median, and distribution).

Another limitation revealed during the application of this protocol is that the number of materials defined is generally proportional to the number of samples in the data. As discussed in Section 4.3.2, it is possible that individual materials defined by this method may not yield different sensor responses. For this reason, it is critical to determine the limitations of the sensors that are being tested using the 3-D models produced via this protocol. In other words, better-defined relationships between soil properties and sensor response, including specific information about sensor fidelity, would allow users to set the protocol threshold to a value that is realistic and effective for a given sensor. Knowledge of sensor fidelity may allow for very fine adjustment of the protocol, such that the protocol may be able to delineate soil variability at many different scales. Accurate "sub-materials" may be definable with a very small threshold value. Likewise, larger "super-materials" may be defined to characterize macro-scale geologic features. 


\section{Conclusion}

The GEOTACS Subsurface Realizations team successfully developed a prototype quantitative method to define soil materials for 3-D modeling of near-surface geology. This method was developed as one component of 3-D geo-environmental modeling used in force protection and is an integral component of current and future modeling efforts for sensor response simulation. Although the protocol is limited by unavoidable soil-modeling complexities, it offers a number of promising developments over previous methods and is adaptable to ever-changing tactical objectives. The development process also revealed important complexities about classifying soil materials by using properties known to affect sensor response.

\subsection{Adaptable improvements on previous methods}

To build a geological framework for near-surface models, it is essential to define material types according to the intent of the model. The system presented here uses statistical methods to define material types by using properties affecting sensor response. Earlier methods hinged on qualitative interpretations that met the need of the time but might not have been reliably repeatable. The magnitude of the threshold could be adjusted based on the sensor system being modeled, and in theory an array of threshold values could be used as needed for each parameter being evaluated.

In this study, threshold values were defined by trial and error. Experiments with several threshold values revealed that the approach could be tailored to the scale of the model. For a given sensor, the threshold value may be relatively large, meaning the sensor is capable of seeing only large-scale differences in a property rather than small differences in different soils. On the other hand, smaller threshold values can reveal variability within one material type. This ability to scale the variability will be critical for advancing the method to serve multiple geo-environments and sensor modalities.

Early methods did not have the advantage of robust and repeatable quantitative tools to perform material classification analysis. Instead, they relied heavily on expert judgments and field observations (i.e., qualitative data). Because sensors measure the near surface using a variety of instrumentation and the sensor-response data are therefore quantitative 
in nature, quantitative tools are bound to more closely reflect the nuances of sensor response models.

The list of parameters used in the prototype method was derived from the results of numerous studies regarding soil-sensor interaction. It is challenging to classify soils or define soil material types on the basis of properties that impact signal attenuation when:

1. Controllers of signal attenuation are not uniquely defined;

2. Relationships among properties are not quantified; and

3. Different sensors are affected by different properties.

Thus, there is no generic all-purpose property and no generic sensor. In light of the deficiencies in the state-of-knowledge about soil-sensor interaction, the protocol presented here is a simple solution that is in its infancy. Improvements in differentiating among soil materials will therefore depend on future research to determine how the various properties that impact sensor response interact with each other and to what extent those interactions affect sensor signals.

An unintended benefit of this research was renewed interest in developing advanced capabilities for TPROGS for GMS. Currently, TPROGS can handle only five material types. While it is important to avoid developing models that are more complex than necessary, the five-material restriction reduces the versatility of a software package that otherwise meets the immediate need. Moreover, the variability of scales and sensor fidelities projected to be needed for GEOTACS simulations will likely require a large number of soil materials to be modeled at a sub-centimeter scale.

\subsection{Quantitative soil parameter comparisons}

The protocol uses the standard, or Z-score, method to compare soil parameter data. Most such parametric statistical techniques require the assumption that the data are normally distributed (i.e., Gaussian). Nonparametric statistics do not carry the same assumption of normality, but they often have a bottom limit to the number of data points required to yield statistically significant results (i.e., confidence interval greater than 95 percent). In this case, the total dataset (consisting of one soil parameter for multiple sites and multiple depths) was large, the sub-datasets (consisting of single-parameter and site groups) were small, and most of the data and sub-datasets were not normally distributed. So, while the Z-score method 
employed in the protocol is a valid first-order approach to the problem, it also suggests that additional work is required to identify the most realistic and effective statistical method.

The quantitative component of the protocol also revealed some important things about the soil parameters used. First, certain aspects of the USCS classification system were found to be useful outside civil engineering applications. The coefficient of uniformity $\left(\mathrm{C}_{\mathrm{u}}\right)$, for example, was used in a way other than originally intended. By standard USCS methods, soils are considered to be poorly graded if their $\mathrm{C}_{\mathrm{u}}$ is greater than six and their $\mathrm{C}_{\mathrm{c}}$ is greater than three. These values are realistic for sands and gravels that were engineered for use in roadway construction. Soils of natural geologic settings are not processed and thus are likely to be poorly graded. Large values of $\mathrm{C}_{\mathrm{u}}$ for natural soils indicate extremely wide ranges of particle sizes in a given soil. Since particle size can impact sensor response, $\mathrm{C}_{\mathrm{u}}$ can be used as a quantitative parameter to distinguish natural material types for sensor-response simulations. The same is also true for the coefficient of curvature, $\mathrm{C}_{\mathrm{c}}$.

Second, the results of our protocol tests revealed some new information about correlations between various soil properties. Dry bulk density $(\rho)$ and porosity $(\varphi)$, for example, consistently showed similar materials definitions according to their Z-scores. This was an expected (albeit not guaranteed) relationship. On the other hand, the materials definition from specific conductance $(\sigma)$ and effective saturation (Se) were not as similar as expected. These results are preliminary but can be used in future work to develop and support the examination of cross-correlations between soil properties.

\subsection{Future research}

The primary goal for future research will be to design and implement a statistical method that increases the number of soil-property correlations that are directly accounted for during analyses. We also intend to explore the application of non-parametric statistics and high-performance computing. Respectively, these tools may provide more robust analyses by eliminating assumptions of data normality and providing the computing power for the large numbers of calculations associated with "brute force" statistics. 


\section{References}

American Association of State Highway and Transportation Officials. 2010. Standard specifications for transportation materials and methods of sampling and testing 3oth ed. Washington, DC: American Association of State Highway and Transportation Officials.

2010. AASHTO provisional standards. Washington, DC: American Association of State Highway and Transportation Officials.

American Society for Testing and Materials. 2010. Standard test methods for apparent density, bulk factor, and pourability of plastic materials . Designation: D1895 96. West Conshohocken, PA: ASTM International.

2010. Standard practice for classification of soils for engineering purposes. Designation: D2487 10. West Conshohocken, PA: ASTM International.

2008. Standard test methods for determination of the soil water chararcteristic curve for desorption using a hanging column, pressure extractor, chilled mirror hygrometer, and/or centrifuge. Designation: D6836 02. West Conshohocken, PA: ASTM International.

Andraski, B. 1996. Properties and variability of soil and trench fill at an arid waste-burial site. Soil Science Society of America Journal 60: 54-56.

Arcone, S., S. Grant, G. Boitnott, and B. Bostick. 2008. Complex permittivity and clay mineralogy of grain-size fractions in a wet silt soil. Geophysics 73 (3): 1-13.

Boadu, F. 2000. Hydraulic conductivity of soils from grain-size distribution: New models. Journal of Geotechnical and Geoenvironmental Engineering 126 (8): 739-46.

Chan, C., and R. Knight. 1999. Determining water content and saturation from dielectric measurements in layered materials. Water Resources Research 35: 85-93.

Curtis, J. 2001. Moisture effects on the dialelectric properties of soils. Transactions in Geoscience and Remote Sensing 39 (1): 125-28.

Davis, J. 2002. Statistics and data analysis in geology. New York, NY: John Wiley \& Sons.

Eslinger, O., A. Hines, S. Howington, J. Ballard, J. Peters, B. White, and P. McAllister. 2007. Omicron: Rapid mesh generation on HPC platforms for the study of near surface phenomena with remote sensing. Proceedings of the HPCMP Users Group Conference. Pittsburgh, PA: IEEE Computer Society.

Fairley, J., S. Price, R. Kala, J. Ballard, L. Wakeley, E. Berney, S. E. Howington, R. A. Goodson, O. J. Eslinger, and A. M. Hines. 2010. Understanding geophysical processes that affect signatures in the electromagnetic spectrum through the use of geo-environmental modeling. Proceedings of the 27th Annual Army Science Conference. Orlando, FL: U.S. Army ASAALT. 
Fredlund, D. G., and A. Xing. 1994. Equation for the soil-water characteristic curve. Canadian Geotechnical Journal 31(3):521-32.

Horner, D. 2009. Program review presentation of the Geo-Environmental Tactical Sensor Simulation (GEOTACS) research program: R.IS.2009.06. Vicksburg, MS: U. S. Army Research and Development Center.

Howari, F., M. Sherif, V. Singh, and M. Al Asam. 2007. Application of GIS and Remote Sensing Techniques in identification, assessment and development of groundwater resources. In Groundwater: Resource evaluation, augmentation, contamination, restoration, modeling and management. Edited by M. Thangaranjan . New Delhi, India: Capital Publishing Company. 1-25.

Howington, S., O. Eslinger, J. Hensley, A. Hines, J. Ballard, M. Farthing, and J. R. Fairley. 2010. The role of hydrogeology in remote sensing for threat detection. Proceedings of the 2010 Army Science Conference. Orlando, FL.

Howington, S., J. Peters, J. Ballard, O. Eslinger, J. Fairley, R. Kala, R.A. Goodson, S. J. Price, A. M. Hines, L. D. and Wakeley. 2011. Using computer simulation to explore the importance of hydrogeology in remote sensing for explosive threat detection. In Military aspects of hydrogeology: Past and present. Edited by J.A. Mather. London: Geological Society of London.

Jackson, S. 2009. Research methods and statistics: A critical thinking approach 3rd ed. Belmont, CA: Wadsworth Cengage Learning.

Jensen, J. 2000. Remote sensing of the environment: An earth resource perspective. Upper Saddle River, NJ: Prentice Hall.

Koh, G., and L. Wakeley. 2009. Attenuation of electromagnetic energy in soil from Yuma Proving Ground. Technical Report TR-09-9. Vicksburg, MS: U.S. Army Engineer Research and Development Center.

Koh, G., and L. Wakeley. 2010. Effect of moisture on radar attenuation in desert soils. Proceedings of the 27th Annual Army Science Conference. Orlando, FL: U.S. Army ASAALT.

Massart, D., J. Smeyers-Verbeke, X. Capron, and K. Schlesier. 2005. Practical data handling: Visual presentation of data by means of box plots. LC GC Europe 18 (4): 215-218:http://www.lcgceurope.com/lcgceurope/data/articlestandard/ lcgceurope/132005/152912/article.pdf. (Accessed 13 May 2011).

Mathews, P. 2005. Design of experiments with Minitab. Milwaukee, WI: ASQ Quality Press.

Moysey, S., and R. Knight. 2004. Modeling the field-scale relationship between dielectric constant and water content in heterogeneous systems. Water Resources Research 40, Wo3510, doi:10.1029/2003WRo02589.

Mualem, Y. 1976. A new model predicting the hydraulic conductivity of unsaturated porous media. Water Resources Research 12 (3): 513-522, doi:10.1029/WR012ioo3poo513. 
Peters, J., S. Howington, O. Eslinger, J. Fairley, J. Ballard, R. Goodson, and V. Carpenter. 2007. Signature evaluation for thermal infrared countermine and IED detection systems. Proceedings of the 2007 DoD High Performance Computing Modernization Program Users Group Conference. Washington, D.C.: IEEE Computer Society. 238-46.

Prothero, D., and F. Schwab. 1996. Sedimentary geology: An introduction to sedimentary rocks and stratigraphy. New York: W.H. Freeman and Company.

Rechenmacher, A., and Z. Medina-Cetina. 2007. Calibration of soil constitutive models with spatially varying parameters. Journal of Geotechnical and Geoenvironmental Engineering 133 (12): 1567-76.

Robinson, D., S. Jones, J. Wraith, D. Or, and S. Friedman. 2003. Advances in dielectric and electrical conductivity measurement using time domain reflectometry: Simultaneous measurement of water content and bulk electrical conductivity in soils and porous media. Vadose Zone Journal 2: 444-75.

Roth, C., M. Malicki, and R. Plagge. 1992. Empirical evaluation of the relationship between soil dielectric constant and volumetric water content as the basis for calibrating soil moisture measurements by TDR. European Journal of Soil Science 13: 1-13.

Soil Survey Staff. 2006. Keys to Soil Taxonomy. 10th ed. Washington, DC: USDA-Natural Resources Conservation Service.

StatSoft. 2010. Electronic statistics textbook. Tulsa, OK: StatSoft, Inc. Retrieved from http://www.statsoft.com/textbook/ (Accessed 13 May 2011).

Talbot, C., C. Hansen, and E. Edris. 2003. New modeling tools for stratigraphic and stochastic modeling and uncertainty analysis. Proceedings of the 2003 Watershed Systems Conference. Portland, OR: U.S. Army Corps of Engineers. 110.

Thevanayagan, S. 1995. Frequency-domain analysis of electrical dispersion of soils. Journal of Geotechnical Engineering 121 (8): 618-28.

Turner, K., M. Hudson, K. Murray, and D. Mott. 2007. Three-dimensional geologic framework model for a karst aquifer system, Hasty and Western Grove quandrangles, northeastern Arkansas. Scientific Investigations Report 20075095. Denver, CO: U.S. Geological Survey.

U.S. Army Corps of Engineers. 1960. The unified soil classification system. Technical Memorandum No. 3-357. Vicksburg, MS: U.S. Army Engineer Waterways Experiment Station.

Van Dam, R., B. Borchers, and J. Hendrickx. 2005. Methods for prediction of soil dielectric properties: a review. Detection and remediation technologies for mines and minelike targets $X .5794$. 188-97.

Van Genuchten, M. 1980. A closed-form equation for predicting the hydraulic conductivity of unsaturated soils. Soils Science Society of America Journal 44: 892-98. 
Wakeley, L. D., S. W. Broadfoot, B. D. Haugen, S. G. Bourne, C. A. Talbot, J. R. Kelley, S. S. Jackson, C. Roig-Silva, and J. R. McKenna. 2011. Distributing and modeling soil properties in a near-surface geologic environment. Technical Report TR-119. Vicksburg, MS: U.S. Army Engineer Research and Development Center.

Wakeley, L., S. Broadfoot, C. Roig-Silva, B. Haugen, S. Bourne, and C. Talbot C. 2010. Defining 3-D geologic architecture and soil variability for sensor simulations. Proceedings of the 27th Army Science. Conference. Orlando, FL: U.S. Army ASSALT.

Wakeley, L., J. Kelley, T. Berry, S. Jackson, and S. Bourne. 2009. Protocol for field sampling of soils to support modeling of geo-environments and soil-sensor interaction. Technical Report TR-09-7. Vicksburg, MS: U.S. Army Engineer Research and Development Center.

Wakeley, L., J. Kelley, C. Talbot, M. Pearson, and S. Broadfoot. 2007. Geologic conceptual model of Mosul Dam. Technical Report TR-07-06. Vicksburg, MS: U.S. Army Engineer Research and Development Center.

Weissmann, G. 1999. Toward new models of subsurface heterogeneity: An alluvial fan sequence stratigraphic framework with transition probability geostatistics. University of California at Davis. Ann Arbor: UMI Dissertation Services. 


\section{Appendix A: Raw Values of Soil Parameters at Each Protocol Test Site}

\section{Site B}

\begin{tabular}{|c|c|c|c|c|c|c|c|c|c|c|c|c|c|c|c|}
\hline Sample & ASTM Classification & $\mathrm{Cu}$ & $\operatorname{std} \mathrm{Cu}$ & Cc & $\operatorname{std} \mathrm{Cc}$ & $\phi$ & $\phi$ std & $\rho$ & $\rho$ std & $\sigma$ & $\sigma$ std & $\mathbf{R}$ & Rstd & $\alpha$ & $\alpha$ std \\
\hline site B 0-1,2 & Silty sand (SM) & 5.556 & -1.210 & 0.747 & $\mid-1.178$ & 37.544 & 0.214 & 1.655 & $\mid-0.020$ & 190.000 & 0.023 & 3.342 & 2.292 & 0.199 & -1.500 \\
\hline site B-10-1,2 & Silty sand (SM) & 14.444 & 0.255 & 1.617 & 1.273 & 38.321 & 0.505 & 1.628 & $|-0.407|$ & 120.000 & $\mid-0.613$ & 2.648 & 0.867 & 0.247 & -0.857 \\
\hline site B-31-1,2 & Silty sand (SM) & 19.524 & 1.092 & 1.672 & 1.429 & 39.500 & \begin{tabular}{|l|}
0.947 \\
\end{tabular} & 1.597 & $|-0.857|$ & 140.000 & \begin{tabular}{|l|}
-0.431 \\
\end{tabular} & 1.861 & -0.747 & 0.349 & 0.494 \\
\hline site B-50-1,3 & Silty sand (SM) & 24.286 & 1.878 & 1.213 & 0.136 & 40.428 & 1.295 & 1.561 & $\mid-1.384$ & 95.000 & \begin{tabular}{|c|}
-0.841 \\
\end{tabular} & 1.436 & -1.619 & 0.421 & 1.441 \\
\hline site B-70-1,3 & Well-graded sand with silt and gravel (SW-SM)g & 20.000 & 1.171 & 1.328 & 0.459 & 36.739 & $\mid-0.089$ & 1.645 & $\mid-0.169$ & 99.000 & $\mid-0.804$ & 2.052 & -0.355 & 0.366 & 0.713 \\
\hline site B-90-1,2 & Well-graded sand with silt and gravel (SW-SM)g & 16.000 & 0.512 & 1.778 & 1.725 & 40.936 & 1.486 & 1.553 & -1.491 & 95.000 & -0.841 & 2.340 & 0.237 & 0.368 & 0.746 \\
\hline site B-100-2,3 & Poorly-graded sand (SP) & 11.765 & -0.187 & 0.795 & -1.042 & 34.407 & -0.963 & 1.719 & 0.898 & 170.000 & -0.158 & 2.216 & -0.019 & 0.279 & -0.433 \\
\hline site B-110-1,2 & Poorly-graded sand (SP) & 10.000 & -0.478 & 0.940 & -0.633 & 34.297 & -1.004 & 1.721 & 0.939 & 120.000 & -0.613 & 2.177 & -0.098 & 0.285 & -0.360 \\
\hline site B-130-2,3 & Poorly-graded sand (SP) & 7.368 & -0.911 & 0.903 & -0.740 & 33.496 & -1.305 & 1.756 & 1.436 & 180.000 & $\mid-0.067$ & 2.647 & 0.866 & 0.220 & -1.222 \\
\hline site B-150-1,3 & Poorly-graded sand (SP) & 8.500 & \begin{tabular}{|l|}
-0.725 \\
\end{tabular} & 0.989 & -0.495 & 34.227 & -1.030 & 1.723 & 0.966 & 440.000 & 2.297 & 2.161 & -0.132 & 0.259 & -0.705 \\
\hline site B-170-2,3 & Poorly-graded sand (SP) & 11.765 & \begin{tabular}{|l|}
-0.187 \\
\end{tabular} & 0.956 & -0.590 & 38.975 & 0.750 & 1.605 & \begin{tabular}{|c|}
-0.745 \\
\end{tabular} & 240.000 & 0.478 & 1.956 & -0.552 & 0.426 & 1.514 \\
\hline site B-190-1,2 & Poorly-graded sand (SP) & 5.556 & -1.210 & 1.043 & -0.344 & 34.826 & -0.806 & 1.714 & \begin{tabular}{|l|}
0.834 \\
\end{tabular} & 360.000 & 1.570 & 1.864 & -0.741 & 0.325 & 0.169 \\
\hline
\end{tabular}

\begin{tabular}{|c|c|c|c|c|c|c|c|c|c|c|}
\hline \\
\hline \multicolumn{11}{|c|}{\begin{tabular}{|l|} 
CBU data (cont.) \\
Sample
\end{tabular}} \\
\hline site B 0-1,2 & 59.000 & -0.268 & 14.000 & \begin{tabular}{|l|}
-0.373 \\
\end{tabular} & 6.000 & -1.815 & 14.000 & 2.233 & 8.000 & 0.914 \\
\hline site B -10-1,2 & 60.000 & -0.071 & 10.000 & -1.684 & 8.000 & -1.054 & 2.000 & -1.318 & 11.000 & 1.764 \\
\hline site B-31-1,2 & 53.000 & -1.447 & 19.000 & 1.267 & 8.000 & -1.054 & 6.000 & -0.134 & 10.000 & 1.480 \\
\hline site B-50-1,3 & 60.000 & -0.071 & 13.000 & -0.700 & 14.000 & 1.227 & 8.000 & 0.457 & 5.000 & 0.064 \\
\hline site $\mathrm{B}-70-1,3$ & 57.500 & -0.563 & 15.500 & \begin{tabular}{|l|}
0.119 \\
\end{tabular} & 10.500 & -0.104 & 7.000 & 0.161 & 6.000 & 0.348 \\
\hline site B-90-1,2 & 61.000 & 0.125 & 15.000 & -0.045 & 10.500 & -0.104 & 9.500 & 0.901 & 3.000 & -0.502 \\
\hline site B-100-2,3 & 58.500 & -0.366 & 20.000 & 1.594 & 10.500 & -0.104 & 7.500 & 0.309 & 1.500 & -0.927 \\
\hline site B-110-1,2 & N/A & $\mathrm{N} / \mathrm{A}$ & N/A & $\mathrm{N} / \mathrm{A}$ & N/A & $\mathrm{N} / \mathrm{A}$ & $\mathrm{N} / \mathrm{A}$ & $\mathrm{N} / \mathrm{A}$ & $\mathrm{N} / \mathrm{A}$ & $\mathrm{N} / \mathrm{A}$ \\
\hline site B-130-2,3 & 67.000 & 1.304 & 13.000 & -0.700 & 13.000 & 0.847 & 6.000 & -0.134 & 2.000 & -0.785 \\
\hline site B-150-1,3 & 70.000 & 1.893 & 14.000 & -0.373 & 11.000 & 0.086 & 3.000 & -1.022 & 2.000 & -0.785 \\
\hline site B-170-2,3 & 64.000 & 0.714 & 14.000 & -0.373 & 13.000 & 0.847 & 4.000 & -0.726 & 3.000 & -0.502 \\
\hline site B-190-1,2 & 54.000 & -1.250 & \begin{tabular}{|l|}
19.000 \\
\end{tabular} & \begin{tabular}{|l|}
1.267 \\
\end{tabular} & \begin{tabular}{|l|}
14.000 \\
\end{tabular} & 1.227 & 4.000 & -0.726 & 1.000 & -1.068 \\
\hline
\end{tabular}




\section{CUL-2}

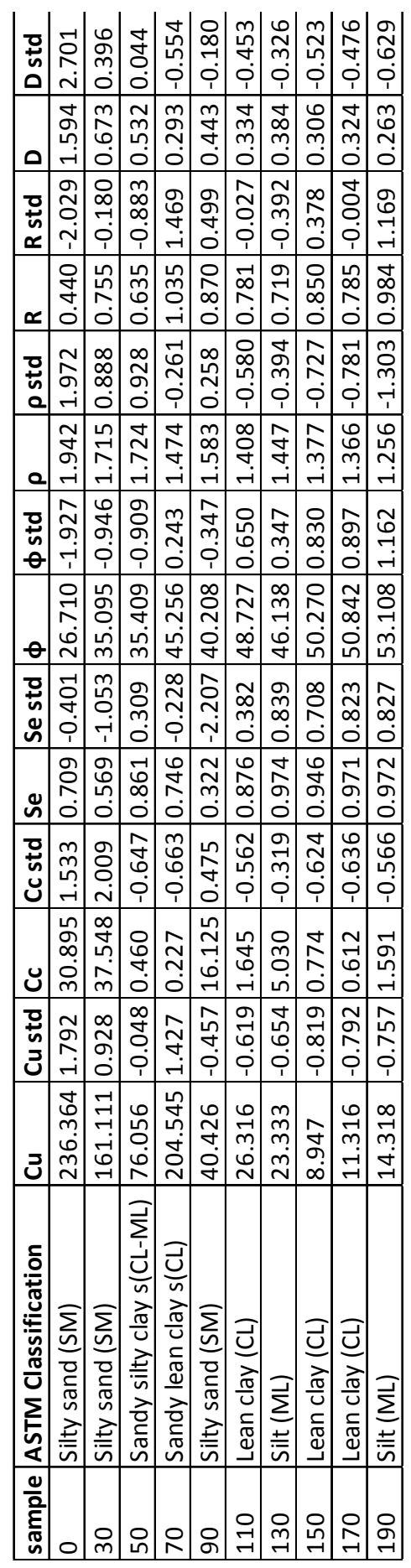


GA1

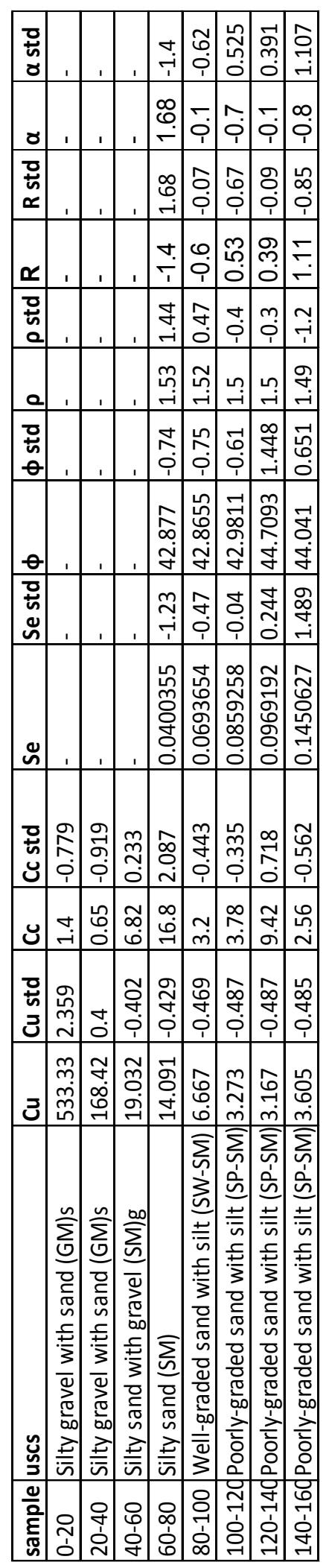


GQ1

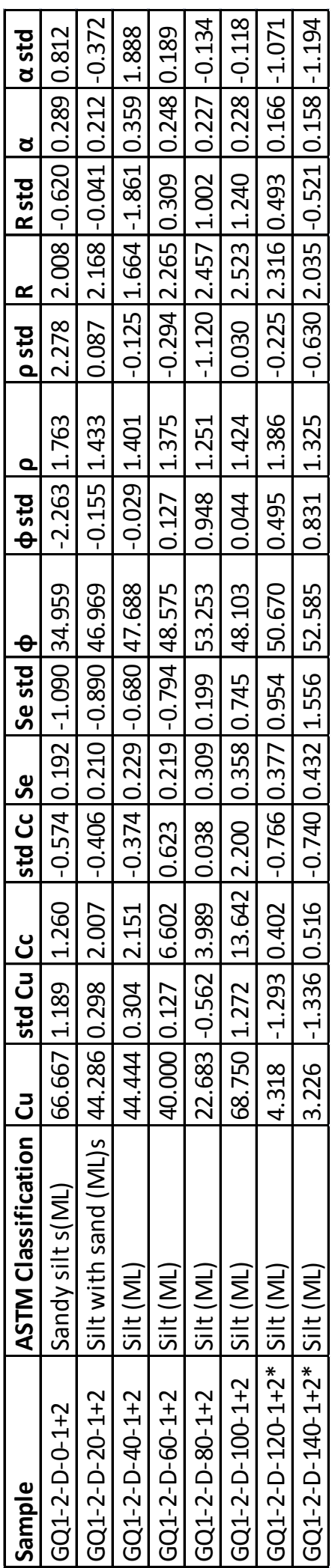




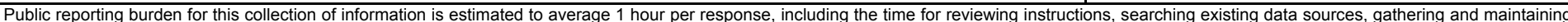

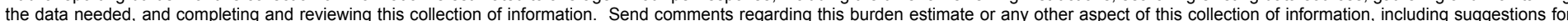

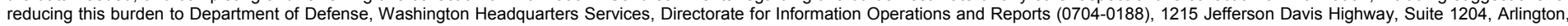

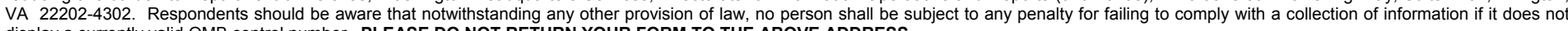
display a currently valid OMB control number. PLEASE DO NOT RETURN YOUR FORM TO THE ABOVE ADDRESS.
1. REPORT DATE (DD-MM-YYYY)
March 2012
Final Report

\section{TITLE AND SUBTITLE}

Defining Soil Materials for 3-D Models of the Near Surface

\section{DATES COVERED (From - To)}

5a. CONTRACT NUMBER

5c. PROGRAM ELEMENT NUMBER

\section{AUTHOR(S)}

Carla Roig-Silva, Benjamin D. Haugen, and Lillian D. Wakeley

5d. PROJECT NUMBER

5e. TASK NUMBER

5f. WORK UNIT NUMBER

\section{PERFORMING ORGANIZATION NAME(S) AND ADDRESS(ES)}

8. PERFORMING ORGANIZATION REPORT NUMBER

Geotechnical and Structures Laboratory

U.S. Army Engineer Research and Development Center

ERDC/GSL TR-12-9

3909 Halls Ferry Road

Vicksburg, MS 39180-6199

\section{SPONSORING / MONITORING AGENCY NAME(S) AND ADDRESS(ES)}

U.S. Army Corps of Engineers

441 G Street NW

Washington, DC 20314-1000

\section{DISTRIBUTION / AVAILABILITY STATEMENT}

Approved for public release; distribution is unlimited.

\section{SUPPLEMENTARY NOTES}

\section{ABSTRACT}

In support of three-dimensional modeling of soils in the near surface, a method was developed to define soil material types quantitatively using statistical comparisons of properties expected to impact sensor performance. To maintain technical continuity with other soils research and ensure relevance to soils engineering, many of the parameters chosen for statistical comparisons included conventional properties familiar to geotechnical researchers. Other, more sensor-specific soil properties, such as effective saturation and thermal conductivity, were used to allow for direct correlation between classified soil materials and sensor response. Initial trials of the method evaluated datasets from four sites in the U.S. and Asia. Early results showed that the number of statistically distinguishable materials tends to mimic the number of soil horizons sampled for a given dataset. Results also revealed a critical need for further research on the interactions of various soil properties and states between and among each other to determine the combinations that have the strongest influence on sensor response patterns. This protocol for delineating near-surface soil materials advances earlier techniques and improves the state-of-knowledge of modeling geologic features in three dimensions for sensor simulation.

\begin{tabular}{|c|c|c|c|c|c|}
\hline \multicolumn{2}{|l|}{ 15. SUBJECT TERMS } & \multicolumn{2}{|l|}{ GMS } & \multicolumn{2}{|l|}{ Soils } \\
\hline \multicolumn{2}{|l|}{ Geostatistics } & \multicolumn{2}{|l|}{ Shallow subsurface } & \multicolumn{2}{|c|}{ Three-dimensional modeling } \\
\hline \multicolumn{2}{|l|}{ GEOTACS } & \multicolumn{2}{|l|}{ Soil geology } & \multicolumn{2}{|c|}{ T-PROGS } \\
\hline \multicolumn{3}{|c|}{ 16. SECURITY CLASSIFICATION OF: } & $\begin{array}{l}\text { 17. LIMITATION } \\
\text { OF ABSTRACT }\end{array}$ & $\begin{array}{l}\text { 18. NUMBER } \\
\text { OF PAGES }\end{array}$ & $\begin{array}{l}\text { 19a. NAME OF RESPONSIBLE } \\
\text { PERSON: Carla Roig-Silva }\end{array}$ \\
\hline $\begin{array}{c}\text { a. REPORT } \\
\text { UNCLASSIFIED }\end{array}$ & $\begin{array}{c}\text { b. ABSTRACT } \\
\text { UNCLASSIFIED }\end{array}$ & $\begin{array}{c}\text { c. THIS PAGE } \\
\text { UNCLASSIFIED }\end{array}$ & UNCLASSIFIED & 56 & $\begin{array}{l}\text { 19b. TELEPHONE NUMBER (include } \\
\text { area code) } \\
\text { (601) 634-3326 }\end{array}$ \\
\hline
\end{tabular}

